

\title{
Finite descent obstructions and rational points on curves
}

\author{
Michael Stoll
}

Let $k$ be a number field and $X$ a smooth projective $k$-variety. In this paper, we study the information obtainable from descent via torsors under finite $k$-group schemes on the location of the $k$-rational points on $X$ within the adelic points. Our main result is that if a curve $C / k$ maps nontrivially into an abelian variety $A / k$ such that $A(k)$ is finite and $\amalg(k, A)$ has no nontrivial divisible element, then the information coming from finite abelian descent cuts out precisely the rational points of $C$. We conjecture that this is the case for all curves of genus at least 2. We relate finite descent obstructions to the Brauer-Manin obstruction; in particular, we prove that on curves, the Brauer set equals the set cut out by finite abelian descent. Our conjecture therefore implies that the Brauer-Manin obstruction against rational points is the only one on curves.

An errata was posted on 20 March 2017 in an online supplement.

\section{Introduction}

In this paper we explore what can be deduced about the set of rational points on a curve (or a more general variety) from the knowledge of its finite étale coverings.

Given a smooth projective variety $X$ over a number field $k$ and a finite étale, geometrically Galois covering $\pi: Y \rightarrow X$, standard descent theory tells us that there are only finitely many twists $\pi_{j}: Y_{j} \rightarrow X$ of $\pi$ such that $Y_{j}$ has points everywhere locally, and then $X(k)=\bigsqcup_{j} \pi_{j}\left(Y_{j}(k)\right)$. Since $X(k)$ embeds into the adelic points $X\left(\mathbb{A}_{k}\right)$, we obtain restrictions on where the rational points on $X$ can be located inside $X\left(\mathbb{A}_{k}\right)$, that is, we must have

$$
X(k) \subset \bigcup_{j} \pi_{j}\left(D_{j}\left(\mathbb{A}_{k}\right)\right)=: X\left(\mathbb{A}_{k}\right)^{\pi} .
$$

Putting the information from all such finite étale coverings together, we arrive at

$$
X\left(\mathbb{A}_{k}\right)^{\mathrm{f}-\mathrm{cov}}=\bigcap_{\pi} X\left(\mathbb{A}_{k}\right)^{\pi} .
$$

MSC2000: primary 11G30; secondary $14 \mathrm{G} 05,11 \mathrm{G} 10,14 \mathrm{H} 30$.

Keywords: rational point, descent obstruction, covering, twist, torsor under finite group scheme,

Brauer-Manin obstruction. 
Since the information we get cannot tell us more than on which connected component a point lies at the infinite places, we make a slight modification by replacing the $v$-adic component of $X\left(\mathbb{A}_{k}\right)$ with its set of connected components, for infinite places $v$. In this way, we obtain $X\left(\mathbb{A}_{k}\right)$ • and $X\left(\mathbb{A}_{k}\right)_{\bullet}^{\mathrm{f}-\text { cov }}$.

We can be more restrictive in the kind of coverings we allow. We denote the set cut out by restrictions coming from finite abelian coverings only by $X\left(\mathbb{A}_{k}\right)_{\text {a }}^{\mathrm{f} \text {-ab }}$ and the set cut out by solvable coverings by $X\left(\mathbb{A}_{k}\right)^{\mathrm{f}-\mathrm{sol}}$. Then we have the chain of inclusions

$$
X(k) \subset \overline{X(k)} \subset X\left(\mathbb{A}_{k}\right)_{\bullet}^{\mathrm{f}-\operatorname{cov}} \subset X\left(\mathbb{A}_{k}\right)_{\bullet}^{\mathrm{f}-\mathrm{sol}} \subset X\left(\mathbb{A}_{k}\right)_{\bullet}^{\mathrm{f}-\mathrm{ab}} \subset X\left(\mathbb{A}_{k}\right)_{\bullet},
$$

where $\overline{X(k)}$ is the topological closure of $X(k)$ in $X\left(\mathbb{A}_{k}\right)_{\bullet}$; see Section 5 below.

It turns out that the set cut out by the information coming from finite étale abelian coverings on a curve $C$ coincides with the "Brauer set", which is defined using the Brauer group of $C$,

$$
C\left(\mathbb{A}_{k}\right)_{\bullet}^{\mathrm{f}-\mathrm{ab}}=C\left(\mathbb{A}_{k}\right)_{\bullet}^{\mathrm{Br}} .
$$

This follows easily from the descent theory of Colliot-Thélène and Sansuc; see Section 7. It should be noted, however, that this result seems to be new. It says that on curves, all the information coming from torsors under groups of multiplicative type is already obtained from torsors under finite abelian group schemes.

In this way, it becomes possible to study the Brauer-Manin obstruction on curves via finite étale abelian coverings. For example, we provide an alternative proof of the main result in Scharaschkin's thesis [1999] characterizing $C\left(\mathbb{A}_{k}\right)_{\bullet}^{\mathrm{Br}}$ in terms of the topological closure of the Mordell-Weil group in the adelic points of the Jacobian; see Corollary 7.4.

Let us call $X$ "good" if it satisfies $\overline{X(k)}=X\left(\mathbb{A}_{k}\right)_{\bullet}^{\mathrm{f}-\mathrm{cov}}$ and "very good" if it satisfies $\overline{X(k)}=X\left(\mathbb{A}_{k}\right)_{\bullet}^{\mathrm{f}-\mathrm{ab}}$.

Then another consequence is that the Brauer-Manin obstruction is the only obstruction against rational points on a curve that is very good. More precisely, the Brauer-Manin obstruction is the only one against a weak form of weak approximation, namely weak approximation with information at the infinite primes reduced to connected components.

An abelian variety $A / k$ is very good if and only if the divisible subgroup of $\amalg(k, A)$ is trivial. For example, if $A / \mathbb{Q}$ is a modular abelian variety of analytic rank zero, then $A(\mathbb{Q})$ and $\amalg(\mathbb{Q}, A)$ are both finite, and $A$ is very good. A principal homogeneous space $X$ for $A$ such that $X(k)=\varnothing$ is very good if and only if it represents a nondivisible element of $\amalg(k, A)$. See Corollary 6.2 and the text follows it.

The main result of this paper says that if $C / k$ is a curve that has a nonconstant morphism $C \rightarrow X$, where $X$ is (very) good and $X(k)$ is finite, then $C$ is (very) good 
(and $C(k)$ is finite); see Proposition 8.5. This implies that every curve $C / \mathbb{Q}$ whose Jacobian has a nontrivial factor $A$, namely a modular abelian variety of analytic rank zero, is very good; see Theorem 8.6. As an application, we prove that all modular curves $X_{0}(N), X_{1}(N)$ and $X(N)$ (over $\mathbb{Q}$ ) are very good; see Corollary 8.8. For curves without rational points, we have the following corollary.

Corollary. If $C / \mathbb{Q}$ has a nonconstant morphism into a modular abelian variety of analytic rank zero, and if $C(\mathbb{Q})=\varnothing$, then the absence of rational points is explained by the Brauer-Manin obstruction.

This generalizes a result due to Siksek [2004] by removing all assumptions related to the Galois action on the fibers of the morphism over rational points.

The paper is organized as follows. After a preliminary section (Section 2) setting up the notation, we prove in Section 3 some results on abelian varieties, which will be needed later on, but are also interesting in themselves. Then, in Section 4, we review torsors and twists and set up some categories of torsors for later use. Section 5 introduces the sets cut out by finite descent information, as sketched above, and Section 6 relates this to rational points. Next we study the relationship between our sets $X\left(\mathbb{A}_{k}\right)_{\bullet}^{\mathrm{f}-\operatorname{cov} / \mathrm{f}-\mathrm{sol} / \mathrm{f}-\mathrm{ab}}$ and the Brauer set $X\left(\mathbb{A}_{k}\right)_{\bullet}^{\mathrm{Br}}$ together with its variants. This is done in Section 7. We then discuss certain inheritance properties of the notion of being "excellent" (which is stronger than "good") in Section 8. This is then the basis for the conjecture formulated and discussed in Section 9.

\section{Preliminaries}

In the following, $k$ will always denote a number field.

Let $X$ be a smooth projective variety over $k$. We modify the definition of the set of adelic points of $X$ in the following way. ${ }^{1}$

$$
X\left(\mathbb{A}_{k}\right) \bullet=\prod_{v \nmid \infty} X\left(k_{v}\right) \times \prod_{v \mid \infty} \pi_{0}\left(X\left(k_{v}\right)\right) .
$$

In other words, the factors at infinite places $v$ are reduced to the set of connected components of $X\left(k_{v}\right)$. We then have a canonical surjection $X\left(\mathbb{A}_{k}\right) \longrightarrow X\left(\mathbb{A}_{k}\right)$. Note that for a zero-dimensional variety (or reduced finite scheme) $Z$, we have $Z\left(\mathbb{A}_{k}\right)=Z\left(\mathbb{A}_{k}\right)_{\text {. }}$. We will occasionally be a bit sloppy in our notation, pretending that canonical maps like $X\left(\mathbb{A}_{k}\right)_{\bullet} \rightarrow X\left(\mathbb{A}_{K}\right)$ • (for a finite extension $K \supset k$ ) or $Y\left(\mathbb{A}_{k}\right)_{\bullet} \rightarrow X\left(\mathbb{A}_{k}\right)_{\bullet}$ (for a subvariety $\left.Y \subset X\right)$ are inclusions, even though they in general are not at the infinite places. For example, the intersection $X(K) \cap X\left(\mathbb{A}_{k}\right)$. means the intersection of the images of both sets in $X\left(\mathbb{A}_{K}\right)_{\text {. }}$.

\footnotetext{
${ }^{1}$ This notation was introduced by Bjorn Poonen.
} 
If $X=A$ is an abelian variety over $k$, then

$$
\prod_{v \nmid \infty}\{0\} \times \prod_{v \mid \infty} A\left(k_{v}\right)^{0}=A\left(\mathbb{A}_{k}\right)_{\mathrm{div}}
$$

is exactly the divisible subgroup of $A\left(\mathbb{A}_{k}\right)$. This implies that

$$
A\left(\mathbb{A}_{k}\right)_{\bullet} / n A\left(\mathbb{A}_{k}\right)_{\bullet}=A\left(\mathbb{A}_{k}\right) / n A\left(\mathbb{A}_{k}\right)
$$

and then that

$$
A\left(\mathbb{A}_{k}\right)_{\bullet}=\lim _{\longleftarrow} A\left(\mathbb{A}_{k}\right)_{\bullet} / n A\left(\mathbb{A}_{k}\right)_{\bullet}=\lim _{\longleftarrow} A\left(\mathbb{A}_{k}\right) / n A\left(\mathbb{A}_{k}\right)=\widehat{A\left(\mathbb{A}_{k}\right)}
$$

is (isomorphic to) its own componentwise profinite completion and also the componentwise profinite completion of the usual group of adelic points.

We will denote by $\widehat{A(k)}=A(k) \otimes_{\mathbb{Z}} \hat{\mathbb{Z}}$ the profinite completion $\lim A(k) / n A(k)$ of the Mordell-Weil group $A(k)$. By a result of Serre [1971, Thm. $\overleftarrow{3]}$, the natural map $\widehat{A(k)} \rightarrow \widehat{A\left(\mathbb{A}_{k}\right)}=A\left(\mathbb{A}_{k}\right)$. is an injection and therefore induces an isomorphism with the topological closure $\overline{A(k)}$ of $A(k)$ in $A\left(\mathbb{A}_{k}\right)_{\text {. }}$. We will reprove this in Proposition 3.7 below, and even show something stronger than that; see Theorem 3.10. (Our proof is based on a later result of Serre.) Note that we have an exact sequence

$$
0 \longrightarrow A(k)_{\text {tors }} \longrightarrow \widehat{A(k)} \longrightarrow \hat{\mathbb{Z}}^{r} \longrightarrow 0,
$$

where $r$ is the Mordell-Weil rank of $A(k)$; in particular,

$$
\widehat{A(k})_{\mathrm{tors}}=A(k)_{\mathrm{tors}} \text {. }
$$

Let $\operatorname{Sel}^{(n)}(k, A)$ denote the $n$-Selmer group of $A$ over $k$, as usual sitting in an exact sequence

$$
0 \longrightarrow A(k) / n A(k) \longrightarrow \operatorname{Sel}^{(n)}(k, A) \longrightarrow \amalg(k, A)[n] \longrightarrow 0 .
$$

If $n \mid N$, we have a canonical map of exact sequences

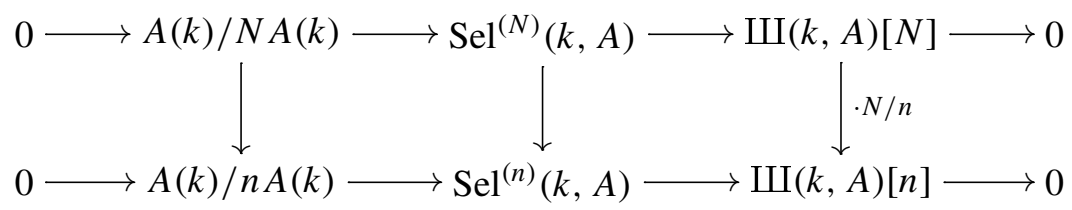

and we can form the projective limit

$$
\widehat{\operatorname{Sel}}(k, A)=\lim _{\longleftarrow} \operatorname{Sel}^{(n)}(k, A),
$$

which sits again in an exact sequence

$$
0 \longrightarrow \widehat{A(k)} \longrightarrow \widehat{\operatorname{Sel}}(k, A) \longrightarrow T \amalg(k, A) \longrightarrow 0,
$$


where $T \amalg(k, A)$ is the Tate module of $\amalg(k, A)$ (and the exactness on the right follows from the fact that the maps $A(k) / N A(k) \rightarrow A(k) / n A(k)$ are surjective). If $\amalg(k, A)$ is finite, or more generally, if the divisible subgroup $\amalg(k, A)_{\text {div }}$ is trivial, then the Tate module vanishes, and $\widehat{\operatorname{Sel}}(k, A)=\widehat{A(k)}$. Note also that since $T \amalg(k, A)$ is torsion-free, we have

$$
\left.\widehat{\operatorname{Sel}}(k, A)_{\text {tors }}=\widehat{A(k}\right)_{\text {tors }}=A(k)_{\text {tors }} .
$$

By the definition of the Selmer group, we get maps

$$
\operatorname{Sel}^{(n)}(k, A) \longrightarrow A\left(\mathbb{A}_{k}\right) / n A\left(\mathbb{A}_{k}\right)=A\left(\mathbb{A}_{k}\right)_{\bullet} / n A\left(\mathbb{A}_{k}\right) \bullet
$$

that are compatible with the projective limit, so we obtain a canonical map

$$
\widehat{\operatorname{Sel}}(k, A) \longrightarrow A\left(\mathbb{A}_{k}\right) \text {. }
$$

through which the map $\widehat{A(k)} \rightarrow A\left(\mathbb{A}_{k}\right)$. factors. We will denote the elements of $\widehat{\operatorname{Sel}}(k, A)$ by $\hat{P}, \hat{Q}$ and the like, and we will write $P_{v}, Q_{v}$ and the like for their images in $A\left(k_{v}\right)$ or $\pi_{0}\left(A\left(k_{v}\right)\right)$, so the map $\widehat{\operatorname{Sel}}(k, A) \rightarrow A\left(\mathbb{A}_{k}\right)$. is specified as $\hat{P} \longmapsto\left(P_{v}\right)_{v}$. (It will turn out that this map is injective; see Proposition 3.7.)

If $X$ is a $k$-variety, then we use notation like $\mathrm{Pic}_{X}, \mathrm{NS}_{X}$, and so forth, to denote the Picard group, Néron-Severi group, and so forth, of $X$ over $\bar{k}$, as a $k$-Galois module.

Finally, we will denote the absolute Galois group of $k$ by $\varphi_{k}$.

\section{Some results on abelian varieties}

In the following, $A$ is an abelian variety over $k$ of dimension $g$. For $N \geq 1$, we set $k_{N}=k(A[N])$ for the $N$-division field, and $k_{\infty}=\bigcup_{N} k_{N}$ for the division field.

The following lemma, based on a result due to Serre on the image of the Galois group in $\operatorname{Aut}\left(A_{\text {tors }}\right)$, forms the basis for the results of this section.

Lemma 3.1. There is some $m \geq 1$ such that $m$ kills all the cohomology groups $H^{1}\left(k_{N} / k, A[N]\right)$.

Proof. By a result of Serre [2000, p. 60], the image of $\varphi_{k}$ in $\operatorname{Aut}\left(A_{\text {tors }}\right)=\mathrm{GL}_{2 g}(\hat{\mathbb{Z}})$ meets the scalars $\hat{\mathbb{Z}}^{\times}$in a subgroup containing $S=\left(\hat{\mathbb{Z}}^{\times}\right)^{d}$ for some $d \geq 1$. We can assume that $d$ is even.

Now we note that in

$$
H^{1}\left(k_{N} / k, A[N]\right) \stackrel{\inf }{\hookrightarrow} H^{1}\left(k_{\infty} / k, A[N]\right) \longrightarrow H^{1}\left(k_{\infty} / k, A_{\text {tors }}\right),
$$

the kernel of the second map is killed by \#A(k) tors. Hence it suffices to show that $H^{1}\left(k_{\infty} / k, A_{\text {tors }}\right)$ is killed by some $m$. 
Let $G=\operatorname{Gal}\left(k_{\infty} / k\right) \subset \mathrm{GL}_{2 g}(\hat{\mathbb{Z}})$, then $S \subset G$ is a normal subgroup. We have the inflation-restriction sequence

$$
H^{1}\left(G / S, A_{\text {tors }}^{S}\right) \longrightarrow H^{1}\left(G, A_{\text {tors }}\right) \longrightarrow H^{1}\left(S, A_{\text {tors }}\right) .
$$

Therefore it suffices to show that there is some integer $D \geq 1$ killing both $A_{\text {tors }}^{S}$ and $H^{1}\left(S, A_{\text {tors }}\right)=H^{1}\left(\left(\hat{\mathbb{Z}}^{\times}\right)^{d}, \mathbb{Q} / \mathbb{Z}\right)^{2 g}$.

For a prime $p$, we define

$$
v_{p}=\min \left\{v_{p}\left(a^{d}-1\right): a \in \mathbb{Z}_{p}^{\times}\right\} .
$$

It is easy to see that when $p$ is odd, we have $v_{p}=0$ if $p-1$ does not divide $d$, and $v_{p}=1+v_{p}(d)$ otherwise. Also, $v_{2}=1$ if $d$ is odd (which we excluded), and $v_{2}=2+v_{2}(d)$ otherwise. In particular,

$$
D=\prod_{p} p^{v_{p}}
$$

is a well-defined positive integer.

We first show that $A_{\text {tors }}^{S}$ is killed by $D$. We have

$$
A_{\text {tors }}^{S}=\left(\bigoplus_{p}\left(\mathbb{Q}_{p} / \mathbb{Z}_{p}\right)^{\left(\mathbb{Z}_{p}^{\times}\right)^{d}}\right)^{2 g}
$$

and for an individual summand, we see that

$$
\begin{aligned}
\left(\mathbb{Q}_{p} / \mathbb{Z}_{p}\right)^{\left(\mathbb{Z}_{p}^{\times}\right)^{d}} & =\left\{x \in \mathbb{Q}_{p} / \mathbb{Z}_{p}:\left(a^{d}-1\right) x=0 \quad \forall a \in \mathbb{Z}_{p}^{\times}\right\} \\
& =\left\{x \in \mathbb{Q}_{p} / \mathbb{Z}_{p}: p^{v_{p}} x=0\right\}
\end{aligned}
$$

is killed by $p^{v_{p}}$, whence the claim.

Now we have to look at $H^{1}\left(S, A_{\text {tors }}\right)$. It suffices to consider $H^{1}\left(\left(\hat{\mathbb{Z}}^{\times}\right)^{d}, \mathbb{Q} / \mathbb{Z}\right)$. We start with

$$
H^{1}\left(\left(\mathbb{Z}_{p}^{\times}\right)^{d}, \mathbb{Q}_{p} / \mathbb{Z}_{p}\right)=0 .
$$

To see this, note that $\left(\mathbb{Z}_{p}^{\times}\right)^{d}$ is procyclic (for odd $p, \mathbb{Z}_{p}^{\times}$is already procyclic; for $p=2, \mathbb{Z}_{2}^{\times}$is $\{ \pm 1\}$ times a procyclic group, and the first factor goes away under exponentiation by $d$, since $d$ was assumed to be even). Let $\alpha \in\left(\mathbb{Z}_{p}^{\times}\right)^{d}$ be a topological generator. By evaluating the cocycles at $\alpha$, we obtain an injection

$$
H^{1}\left(\left(\mathbb{Z}_{p}^{\times}\right)^{d}, \mathbb{Q}_{p} / \mathbb{Z}_{p}\right) \hookrightarrow \frac{\mathbb{Q}_{p} / \mathbb{Z}_{p}}{(\alpha-1)\left(\mathbb{Q}_{p} / \mathbb{Z}_{p}\right)}=\frac{\mathbb{Q}_{p} / \mathbb{Z}_{p}}{p^{v_{p}}\left(\mathbb{Q}_{p} / \mathbb{Z}_{p}\right)}=0
$$

We then can conclude that $H^{1}\left(\left(\hat{\mathbb{Z}}^{\times}\right)^{d}, \mathbb{Q}_{p} / \mathbb{Z}_{p}\right)$ is killed by $p^{v_{p}}$. To see this, write

$$
\left(\hat{\mathbb{Z}}^{\times}\right)^{d}=\left(\mathbb{Z}_{p}^{\times}\right)^{d} \times T,
$$


where $T=\prod_{q \neq p}\left(\mathbb{Z}_{q}^{\times}\right)^{d}$. Then, by inflation-restriction again, there is an exact sequence

$$
0=H^{1}\left(\left(\mathbb{Z}_{p}^{\times}\right)^{d}, \mathbb{Q}_{p} / \mathbb{Z}_{p}\right) \longrightarrow H^{1}\left(\left(\hat{\mathbb{Z}}^{\times}\right)^{d}, \mathbb{Q}_{p} / \mathbb{Z}_{p}\right) \longrightarrow H^{1}\left(T, \mathbb{Q}_{p} / \mathbb{Z}_{p}\right)^{\left(\mathbb{Z}_{p}^{\times}\right)^{d}},
$$

and we have (note that $T$ acts trivially on $\mathbb{Q}_{p} / \mathbb{Z}_{p}$ )

$$
H^{1}\left(T, \mathbb{Q}_{p} / \mathbb{Z}_{p}\right)^{\left(\mathbb{Z}_{p}^{\times}\right)^{d}}=\operatorname{Hom}\left(T,\left(\mathbb{Q}_{p} / \mathbb{Z}_{p}\right)^{\left(\mathbb{Z}_{p}^{\times}\right)^{d}}\right) .
$$

This group is killed by $p^{v_{p}}$, since $\left(\mathbb{Q}_{p} / \mathbb{Z}_{p}\right)^{\left(\mathbb{Z}_{p}^{\times}\right)^{d}}$ is. It follows that

$$
H^{1}\left(\left(\hat{\mathbb{Z}}^{\times}\right)^{d}, \mathbb{Q} / \mathbb{Z}\right)=\bigoplus_{p} H^{1}\left(\left(\hat{\mathbb{Z}}^{\times}\right)^{d}, \mathbb{Q}_{p} / \mathbb{Z}_{p}\right)
$$

is killed by $D=\prod_{p} p^{v_{p}}$.

We therefore find that $H^{1}\left(G, A_{\text {tors }}\right)$ is killed by $D^{2}$, and that $H^{1}\left(k_{N} / k, A[N]\right)$ is killed by $D^{2} \# A(k)_{\text {tors }}$, for all $N$.

Remark 3.2. A similar statement is proved for elliptic curves in [Viada 2003, Prop. 7].

Lemma 3.3. For all positive integers $N$, the map

$$
\operatorname{Sel}^{(N)}(k, A) \longrightarrow \operatorname{Sel}^{(N)}\left(k_{N}, A\right)
$$

has the kernel killed by $m$, where $m$ is the number from Lemma 3.1.

Proof. We have the following commutative and exact diagram.

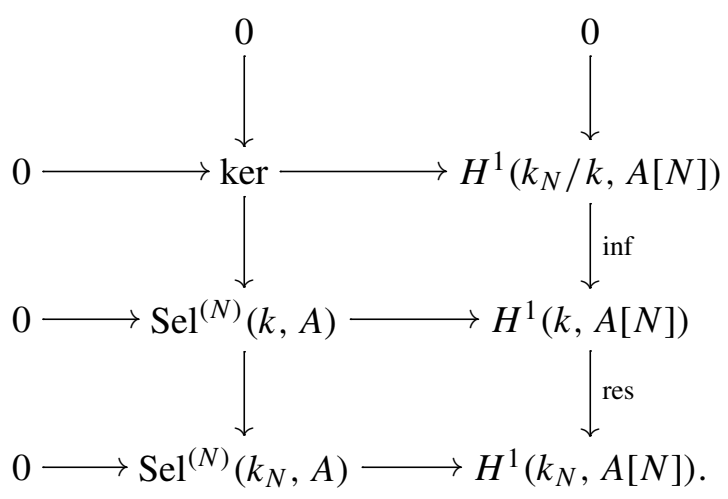

So the kernel in question injects into $H^{1}\left(k_{N} / k, A[N]\right)$, and by Lemma 3.1, this group is killed by $m$.

Lemma 3.4. Let $Q \in \operatorname{Sel}^{(N)}(k, A)$, and let $n$ be the order of $m Q$, where $m$ is the number from Lemma 3.1. Then the density of places $v$ of $k$ such that $v$ splits completely in $k_{N} / k$ and such that the image of $Q$ in $A\left(k_{v}\right) / N A\left(k_{v}\right)$ is trivial is at most $1 /\left(n\left[k_{N}: k\right]\right)$. 
Proof. By Lemma 3.3, the kernel of $\operatorname{Sel}^{(N)}(k, A) \rightarrow \operatorname{Sel}^{(N)}\left(k_{N}, A\right)$ is killed by $m$. Hence the order of the image of $Q$ in $\operatorname{Sel}^{(N)}\left(k_{N}, A\right)$ is a multiple of $n$, the order of $m Q$. Now consider the following diagram for a place $v$ that splits in $k_{N}$ and a place $w$ of $k_{N}$ above it,

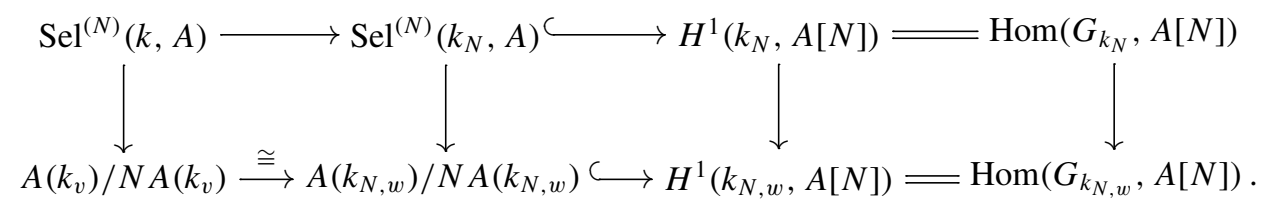

Let $\alpha$ be the image of $Q$ in $\operatorname{Hom}\left(G_{k_{N}}, A[N]\right)$. Then the image of $Q$ is trivial in $A\left(k_{v}\right) / N A\left(k_{v}\right)$ if and only if $\alpha$ restricts to the zero homomorphism on $G_{k_{N, w}}$. This is equivalent to saying that $w$ splits completely in $L / k_{N}$, where $L$ is the fixed field of the kernel of $\alpha$. Since the order of $\alpha$ is a multiple of $n$, we have $\left[L: k_{N}\right] \geq n$, and the claim now follows from the Chebotarev Density Theorem.

Recall the definition of $\widehat{\operatorname{Sel}}(k, A)$ and the natural maps

$$
A(k) \hookrightarrow \widehat{A(k)} \hookrightarrow \widehat{\operatorname{Sel}}(k, A) \longrightarrow A\left(\mathbb{A}_{k}\right) \bullet,
$$

where we denote the rightmost map by

$$
\hat{P} \longmapsto\left(P_{v}\right)_{v} \text {. }
$$

Also recall that $\widehat{\operatorname{Sel}}(k, A)_{\text {tors }}=A(k)_{\text {tors }}$ under the identification given by the inclusions above.

Lemma 3.5. Let $\hat{Q}_{1}, \ldots, \hat{Q}_{s} \in \widehat{\operatorname{Sel}}(k, A)$ be elements of infinite order, and let $n \geq 1$. Then there is some $N$ such that the images of $\hat{Q}_{1}, \ldots, \hat{Q}_{s}$ in $\operatorname{Sel}^{(N)}(k, A)$ all have order at least $n$.

Proof. For a fixed $1 \leq j \leq s$, consider $(n-1) ! \hat{Q}_{j} \neq 0$. There is some $N_{j}$ such that the image of $(n-1) ! \hat{Q}_{j}$ in $\operatorname{Sel}^{\left(N_{j}\right)}(k, A)$ is nonzero. This implies that the image of $\hat{Q}_{j}$ has order at least $n$. Because of the canonical maps $\operatorname{Sel}^{\left(l N_{j}\right)}(k, A) \rightarrow \operatorname{Sel}^{\left(N_{j}\right)}(k, A)$, this will also be true for all multiples of $N_{j}$. Therefore, any $N$ that is a common multiple of all the $N_{j}$ will do.

Proposition 3.6. Let $Z \subset A$ be a finite subscheme of an abelian variety $A$ over $k$ such that $Z(k)=Z(\bar{k})$. Let $\hat{P} \in \widehat{\operatorname{Sel}}(k, A)$ be such that $P_{v} \in Z\left(k_{v}\right)=Z(k)$ for a set of places $v$ of $k$ of density 1 . Then $\hat{P}$ is in the image of $Z(k)$ in $\widehat{\operatorname{Sel}}(k, A)$.

Proof. In the following, we identify $A(k)$ with its image in $\widehat{\operatorname{Sel}}(k, A)$. We first show that $\hat{P} \in Z(k)+A(k)$ tors. Assume the contrary. Then none of the differences $\hat{P}-Q$ for $Q \in Z(k)$ has finite order. Let $n>\# Z(k)$. Then by Lemma 3.5, we can find a number $N$ such that the image of $m(\hat{P}-Q)$ under $\widehat{\operatorname{Sel}}(k, A) \rightarrow \operatorname{Sel}^{(N)}(k, A)$ has order at least $n$, for all $Q \in Z(k)$. 
By Lemma 3.4, the density of places of $k$ such that $v$ splits in $k_{N}$ and at least one of $\hat{P}-Q$, for $Q \in Z(k)$, maps trivially into $A\left(k_{v}\right) / N A\left(k_{v}\right)$ is at most

$$
\frac{\# Z(k)}{n\left[k_{N}: k\right]}<\frac{1}{\left[k_{N}: k\right]} .
$$

Therefore, there is a set of places $v$ of $k$ of positive density such that $v$ splits completely in $k_{N} / k$ and such that none of $\hat{P}-Q$ maps trivially into $A\left(k_{v}\right) / N A\left(k_{v}\right)$. This implies $P_{v} \neq Q$ for all $Q \in Z(k)$, contrary to the assumption on $\hat{P}$ and the fact that $Z\left(k_{v}\right)=Z(k)$.

It therefore follows that $\hat{P} \in Z(k)+A(k)_{\text {tors }} \subset A(k)$. Take a finite place $v$ of $k$ such that $P_{v} \in Z(k)$ (the set of such places has density 1 by assumption). Then $A(k)$ injects into $A\left(k_{v}\right)$. But the image $P_{v}$ of $\hat{P}$ under $\widehat{\operatorname{Sel}}(k, A) \rightarrow A\left(k_{v}\right)$ is in $Z$; therefore we must have $\hat{P} \in Z(k)$.

The following is a simple, but useful consequence.

Proposition 3.7. If $S$ is a set of places of $k$ of density 1 , then

$$
\widehat{\operatorname{Sel}}(k, A) \longrightarrow \prod_{v \in S} A\left(k_{v}\right) / A\left(k_{v}\right)^{0}
$$

is injective. (Note that $A\left(k_{v}\right)^{0}=0$ for $v$ finite.) In particular,

$$
\widehat{A(k)} \longrightarrow \prod_{v \in S} A\left(k_{v}\right) / A\left(k_{v}\right)^{0}
$$

is injective, and the canonical map $\widehat{A(k)} \rightarrow A\left(\mathbb{A}_{k}\right)$. induces an isomorphism between $\widehat{A(k)}$ and $\overline{A(k)}$, the topological closure of $A(k)$ in $A\left(\mathbb{A}_{k}\right)$.

This is essentially Serre's result in [Serre 1971, Thm. 3].

Proof. Let $\hat{P}$ be in the kernel. Then we can apply Proposition 3.6 with $Z=\{0\}$, and we find that $\hat{P}=0$.

In the last statement, it is clear that the image of the map is $\overline{A(k)}$, whence the result.

From now on, we will identify $\widehat{\operatorname{Sel}}(k, A)$ with its image in $A\left(\mathbb{A}_{k}\right)$. We then have a chain of inclusions

$$
A(k) \subset \overline{A(k)} \subset \widehat{\operatorname{Sel}}(k, A) \subset A\left(\mathbb{A}_{k}\right)_{\bullet}, \quad \text { and } \quad \widehat{\operatorname{Sel}}(k, A) / \overline{A(k)} \cong T \amalg(k, A)
$$

vanishes if and only if the divisible subgroup of $\amalg(k, A)$ is trivial.

We can prove a result stronger than the above. For a finite place $v$ of $k$, we denote by $\mathbb{F}_{v}$ the residue class field at $v$. If $v$ is a place of good reduction for $A$, then it makes sense to speak of $A\left(\mathbb{F}_{v}\right)$, the group of $\mathbb{F}_{v}$-points of $A$. There is a canonical map

$$
\widehat{\operatorname{Sel}}(k, A) \longrightarrow A\left(k_{v}\right) \longrightarrow A\left(\mathbb{F}_{v}\right) \text {. }
$$


Lemma 3.8. Let $0 \neq \hat{Q} \in \widehat{\operatorname{Sel}}(k, A)$. Then there is a set of (finite) places $v$ of $k$ (of good reduction for $A$ ) of positive density such that the image of $\hat{Q}$ in $A\left(\mathbb{F}_{v}\right)$ is nontrivial.

Proof. First assume that $\hat{Q} \notin A(k)_{\text {tors }}$. Then $m \hat{Q} \neq 0$, so there is some $N$ such that $m \hat{Q}$ has nontrivial image in $\operatorname{Sel}^{(N)}(k, A)$ (where $m$ is, as usual, the number from Lemma 3.1). By Lemma 3.4, we find that there is a set of places $v$ of $k$ of positive density such that $Q_{v} \notin N A\left(k_{v}\right)$. Excluding the finitely many places dividing $N \infty$ or of bad reduction for $A$ does not change this density. For $v$ in this reduced set, we have $A\left(k_{v}\right) / N A\left(k_{v}\right) \cong A\left(\mathbb{F}_{v}\right) / N A\left(\mathbb{F}_{v}\right)$, and so the image of $\hat{Q}$ in $A\left(\mathbb{F}_{v}\right)$ is not in $N A\left(\mathbb{F}_{v}\right)$, let alone zero.

Now consider the case that $\hat{Q} \in A(k)_{\text {tors }} \backslash\{0\}$. We know that for all but finitely many finite places $v$ of good reduction, $A(k)_{\text {tors }}$ injects into $A\left(\mathbb{F}_{v}\right)$, so in this case, the statement is even true for a set of places of density 1 .

Remark 3.9. Note that the corresponding statement for points $Q \in A(k)$ is trivial; indeed, there are only finitely many finite places $v$ of good reduction such that $Q$ maps trivially into $A\left(\mathbb{F}_{v}\right)$. To see this, consider some projective model of $A$; then $Q$ and 0 are two distinct points in projective space. They will reduce to the same point mod $v$ if and only if $v$ divides certain nonzero numbers $(2 \times 2$ determinants formed with the coordinates of the two points). The lemma above says that things can not go wrong too badly when we replace $A(k)$ by its completion $\widehat{A(k)}$ or even $\widehat{\operatorname{Sel}}(k, A)$.

Theorem 3.10. Let $S$ be a set of finite places of $k$ of good reduction for $A$ and of density 1. Then the canonical homomorphisms

$$
\widehat{\operatorname{Sel}}(k, A) \longrightarrow \prod_{v \in S} A\left(\mathbb{F}_{v}\right) \text { and } \widehat{A(k)} \longrightarrow \prod_{v \in S} A\left(\mathbb{F}_{v}\right)
$$

are injective.

Proof. Let $\hat{Q}$ be in the kernel. If $\hat{Q} \neq 0$, then by Lemma 3.8, there is a set of places $v$ of positive density such that the image of $\hat{Q}$ in $A\left(\mathbb{F}_{v}\right)$ is nonzero, contradicting the assumptions. So $\hat{Q}=0$, and the map is injective.

For applications, it is useful to remove in Proposition 3.6 the requirement that all points of $Z$ have to be defined over $k$.

Theorem 3.11. Let $Z \subset A$ be a finite subscheme of an abelian variety $A$ over $k$. Let $\hat{P} \in \widehat{\operatorname{Sel}}(k, A)$ be such that $P_{v} \in Z\left(k_{v}\right)$ for a set of places $v$ of $k$ of density 1 . Then $\hat{P}$ is in the image of $Z(k)$ in $\widehat{\operatorname{Sel}}(k, A)$.

Proof. Let $K / k$ be a finite extension such that $Z(K)=Z(\bar{k})$. By Proposition 3.6, we have that the image of $\hat{P}$ in $A\left(\mathbb{A}_{K}\right)_{\bullet}$ is in $Z(K)$. Since $\hat{P}$ is $k$-rational, this implies that the image of $\hat{P}$ in $A\left(\mathbb{A}_{K}\right)$. is in $Z(k)$. Now the canonical map 
$A\left(\mathbb{A}_{k}\right)_{\bullet} \rightarrow A\left(\mathbb{A}_{K}\right)_{\bullet}$ is injective except possibly at some of the infinite places, so $P_{v} \in Z(k)$ for all but finitely many places. Now, replacing $Z$ by $Z(k)$ and applying Proposition 3.6 again (this time over $k$ ), we find that $\hat{P} \in Z(k)$, as claimed.

We have seen that for zero-dimensional subvarieties $Z \subset A$, we have

$$
Z\left(\mathbb{A}_{k}\right) \cdot \cap \overline{A(k)}=Z(k),
$$

or even more generally,

$$
Z\left(\mathbb{A}_{k}\right) \bullet \widehat{\operatorname{Sel}}(k, A)=Z(k)
$$

(writing intersections for simplicity). One can ask if this is valid more generally for subvarieties $X \subset A$, that do not contain the translate of an abelian subvariety of positive dimension.

Question 3.12. Is there such a thing as an "Adelic Mordell-Lang Conjecture"?

A possible statement is as follows. Let $A / k$ be an abelian variety and $X \subset A$ a subvariety not containing the translate of a nontrivial subabelian variety of $A$. Then there is a finite subscheme $Z \subset X$ such that

$$
X\left(\mathbb{A}_{k}\right) \bullet \widehat{\operatorname{Sel}}(k, A) \subset Z\left(\mathbb{A}_{k}\right) \bullet .
$$

If this holds, Theorem 3.11 above implies that

$$
X(k) \subset X\left(\mathbb{A}_{k}\right) \bullet \cap \widehat{\operatorname{Sel}}(k, A) \subset Z\left(\mathbb{A}_{k}\right) \bullet \widehat{\operatorname{Sel}}(k, A)=Z(k) \subset X(k)
$$

and therefore $X(k)=X\left(\mathbb{A}_{k}\right) \bullet \widehat{\operatorname{Sel}}(k, A)$. In the notation introduced in Section 5 below and by the discussion in Section 6 , this implies

$$
X(k) \subset X\left(\mathbb{A}_{k}\right)_{\bullet}^{\mathrm{f}-\mathrm{ab}} \subset X\left(\mathbb{A}_{k}\right) \bullet \cap A\left(\mathbb{A}_{k}\right)_{\bullet}^{\mathrm{f}-\mathrm{ab}}=X\left(\mathbb{A}_{k}\right) \bullet \widehat{\operatorname{Sel}}(k, A)=X(k),
$$

and so $X$ is excellent with respect to the abelian coverings (and hence "very good").

Remark 3.13. Note that the Adelic Mordell-Lang Conjecture formulated above is true when $k$ is a global function field, $A$ is ordinary, and $X$ is not defined over $k^{p}$ (where $p$ is the characteristic of $k$ ); see [Voloch 1991]. (The result is also implicit in [Hrushovski 1996].)

\section{Torsors and twists}

We now introduce torsors (under finite étale group schemes) and twists, and describe various constructions that can be done with these objects.

Let $X$ be a smooth projective (reduced, but not necessarily geometrically connected) variety over $k$.

We will consider the following category $\mathscr{C o v}(X)$. Its objects are $X$-torsors $Y$ under $G$ (see for example [Skorobogatov 2001] for definitions), where $G$ is a finite 
étale group scheme over $k$. More concretely, the data consist of a $k$-morphism $\mu: Y \times G \rightarrow Y$ describing a right action of $G$ on $Y$, together with a finite étale $k$ morphism $\pi: Y \rightarrow X$ such that the following diagram is cartesian, id est, identifies $Y \times G$ with the fiber product $Y \times{ }_{X} Y$,

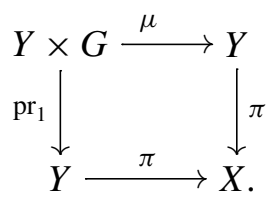

We will usually just write $(Y, G)$ for such an object, with the maps $\mu$ and $\pi$ being understood. Morphisms $\left(Y^{\prime}, G^{\prime}\right) \rightarrow(Y, G)$ in $\mathscr{C o v}(X)$ are given by a pair of maps, as $k$-morphisms of (group) schemes, $\phi: Y^{\prime} \rightarrow Y$ and $\gamma: G^{\prime} \rightarrow G$ such that the obvious diagram

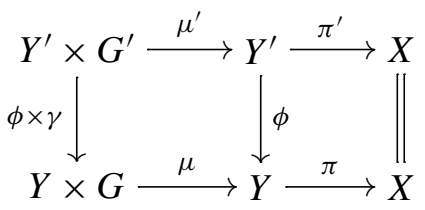

commutes. Note that $\gamma$ is uniquely determined by $\phi$ : if $y^{\prime} \in Y^{\prime}, g^{\prime} \in G^{\prime}$, there is a unique $g \in G$ such that $\phi\left(y^{\prime}\right) \cdot g=\phi\left(y^{\prime} \cdot g^{\prime}\right)$, so we must have $\gamma\left(g^{\prime}\right)=g$.

We will denote by $\mathscr{S o l}(X)$ and $\mathscr{A} b(X)$ the full subcategories of $\mathscr{C o v}(X)$ whose objects are the torsors $(Y, G)$ such that $G$ is solvable or abelian, respectively.

If $X^{\prime} \rightarrow X$ is a $k$-morphism of (smooth projective) varieties, then we can pull back $X$-torsors under $G$ to obtain $X^{\prime}$-torsors under $G$. This defines covariant functors $\mathscr{C o v}(X) \rightarrow \mathscr{C o v}\left(X^{\prime}\right), \mathscr{S o l}(X) \rightarrow \operatorname{Sol}\left(X^{\prime}\right)$ and $\mathscr{A} b(X) \rightarrow \mathscr{A} b\left(X^{\prime}\right)$.

The following constructions are described for $\mathscr{C o v}(X)$, but they are similarly valid for $\mathscr{Y} o l(X)$ and $\mathscr{A} b(X)$.

If $\left(Y_{1}, G_{1}\right),\left(Y_{2}, G_{2}\right) \in \mathscr{C o v}(X)$ are two $X$-torsors, then we can construct their fiber product $(Y, G) \in \mathscr{C o v}(X)$, where $Y=Y_{1} \times_{X} Y_{2}$ and $G=G_{1} \times G_{2}$. More generally, if $\left(Y_{1}, G_{1}\right) \rightarrow(Y, G)$ and $\left(Y_{2}, G_{2}\right) \rightarrow(Y, G)$ are two morphisms in $\mathscr{C o v}(X)$, there is a fiber product $(Z, H) \in \operatorname{Cov}(X)$, where $Z=Y_{1} \times{ }_{Y} Y_{2}$ and $H=G_{1} \times{ }_{G} G_{2}$.

If $(Y, G) \in \operatorname{Cov}(X)$ is an $X$-torsor, where now everything is over $K$ with a finite extension $K / k$, then we can apply restriction of scalars to obtain

$$
\left(R_{K / k} Y, R_{K / k} G\right) \in \mathscr{C o v}\left(R_{K / k} X\right) .
$$

If $(Y, G) \in \operatorname{Cov}(X)$ is an $X$-torsor and $\xi$ is a cohomology class in $H^{1}(k, G)$, then we can construct the twist $\left(Y_{\xi}, G_{\xi}\right)$ of $(Y, G)$ by $\xi$. Here $G_{\xi}$ is the inner form of $G$ corresponding to $\xi$ (compare, for example, [Skorobogatov 2001, pp. 12, 20]). We will denote the structure maps by $\mu_{\xi}$ and $\pi_{\xi}$. Usually, $H^{1}(k, G)$ is just 
a pointed set with distinguished element corresponding to the given torsor; if the torsor is abelian, $H^{1}(k, G)$ is a group, and $G_{\xi}=G$ for all $\xi \in H^{1}(k, G)$.

If $(\phi, \gamma):\left(Y^{\prime}, G^{\prime}\right) \rightarrow(Y, G)$ is a morphism and $\xi \in H^{1}\left(k, G^{\prime}\right)$, then we get an induced morphism

$$
\left(Y_{\xi}^{\prime}, G_{\xi}^{\prime}\right) \rightarrow\left(Y_{\gamma_{*} \xi}, G_{\gamma_{*} \xi}\right)
$$

where $\gamma_{*}$ is the induced map $H^{1}\left(k, G^{\prime}\right) \rightarrow H^{1}(k, G)$. Similarly, twists are compatible with pull-backs, fiber products and restriction of scalars.

Twists are transitive in the following sense. If $(Y, G) \in \mathscr{C o v}(X)$ is an $X$ torsor and $\xi \in H^{1}(k, G), \eta \in H^{1}\left(k, G_{\xi}\right)$, then there is a $\zeta \in H^{1}(k, G)$ such that $\left(\left(Y_{\xi}\right)_{\eta},\left(G_{\xi}\right)_{\eta}\right) \cong\left(Y_{\zeta}, G_{\zeta}\right)$. Conversely, if $\xi$ and $\zeta$ are given, then there is an $\eta \in H^{1}\left(k, G_{\xi}\right)$ such that the relation above holds.

The following observation does not hold in general for $\mathscr{S o l}(X)$ and $\mathscr{A} b(X)$. If $Y \stackrel{\pi}{\rightarrow} X$ is any finite étale morphism, then there is some $(\tilde{Y}, G) \in \mathscr{C o v}(X)$ such that $\tilde{\pi}: \tilde{Y} \rightarrow X$ factors through $\pi$. Also, if we have $(Y, G) \in \mathscr{C o v}(X)$ and $(Z, H) \in$ $\mathscr{C o v}(Y)$, then there is some $(\tilde{Z}, \Gamma) \in \mathscr{C o v}(X)$ such that $\tilde{Z}$ maps to $Z$ over $X$ and such that the induced map $\tilde{Z} \rightarrow Y$ gives rise to a $Y$-torsor $(\tilde{Z}, \tilde{H}) \in \operatorname{Cov}(Y)$. This last statement is also valid with $\mathscr{G o l}(X)$ and $\mathscr{G o l}(Y)$ in place of $\mathscr{C o v}(X)$ and $\mathscr{C o v}(Y)$ (since extensions of solvable groups are solvable).

\section{Finite descent conditions}

In this section, we use torsors and their twists, as described in the previous section, in order to obtain obstructions against rational points. The use of torsors under finite abelian group schemes is classical; it is what is behind the usual descent procedures on elliptic curves or abelian varieties (and so one can claim that they go all the way back to Fermat). The nonabelian case was first studied by Harari and Skorobogatov [2002]; see also [Harari 2000].

The following theorem (going back to Chevalley and Weil [1932]) summarizes the standard facts about descent via torsors. Compare also [Harari and Skorobogatov 2002, Lemma 4.1] and [Skorobogatov 2001, pp. 105, 106].

Theorem 5.1. Let $(Y, G) \in \mathscr{C o v}(X)$ be a torsor, where $X$ is a smooth projective $k$-variety. Then

(1) $X(k)=\bigsqcup_{\xi \in H^{1}(k, G)} \pi_{\xi}\left(Y_{\xi}(k)\right)$;

(2) the $(Y, G)$-Selmer set

$$
\operatorname{Sel}^{(Y, G)}(k, X)=\left\{\xi \in H^{1}(k, G): Y_{\xi}\left(\mathbb{A}_{k}\right) \bullet \neq \varnothing\right\}
$$

is finite: there are only finitely many twists $\left(Y_{\xi}, G_{\xi}\right)$ such that $Y_{\xi}$ has points everywhere locally. 
At least in principle, the Selmer set in the second statement can be determined explicitly, and the union in the first statement can be restricted to this finite set.

The idea behind the following considerations is to see how much information one can get out of the various torsors regarding the image of $X(k)$ in $X\left(\mathbb{A}_{k}\right)$. Compare Definition 4.2 in [Harari and Skorobogatov 2002] and Definition 5.3.1 in Skorobogatov's book Skorobogatov [2001].

Definition 5.2. Let $(Y, G) \in \mathscr{C o v}(X)$ be an $X$-torsor. We say that a point $P \in$ $X\left(\mathbb{A}_{k}\right)_{\bullet}$ survives $(Y, G)$, if it lifts to a point in $Y_{\xi}\left(\mathbb{A}_{k}\right)$. for some twist $\left(Y_{\xi}, G_{\xi}\right)$ of $(Y, G)$.

There is a cohomological description of this property. An $X$-torsor under $G$ is given by an element of $H_{\text {êt }}^{1}(X, G)$. Pull-back through the map Spec $k \rightarrow X$ corresponding to a point in $X(k)$ gives a map

$$
X(k) \longrightarrow H^{1}(k, G) .
$$

Note that it is not necessary to refer to nonabelian étale cohomology here: the map $X(k) \rightarrow H^{1}(k, G)$ induced by a torsor $(Y, G)$ simply arises by associating to a point $P \in X(k)$ its fiber $\pi^{-1}(P) \subset Y$, which is a $k$-torsor under $G$ and therefore corresponds to an element of $H^{1}(k, G)$.

We get a similar map on adelic points,

$$
X\left(\mathbb{A}_{k}\right) \bullet \longrightarrow \prod_{v} H^{1}\left(k_{v}, G\right) .
$$

There is the canonical restriction map

$$
H^{1}(k, G) \longrightarrow \prod_{v} H^{1}\left(k_{v}, G\right),
$$

and the various maps piece together to give a commutative diagram

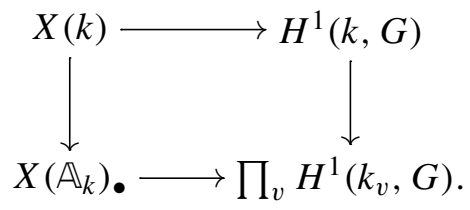

A point $P \in X\left(\mathbb{A}_{k}\right)$ • survives $(Y, G)$ if and only if its image in $\prod_{v} H^{1}\left(k_{v}, G\right)$ is in the image of the global set $H^{1}(k, G)$. The $(Y, G)$-Selmer set is then the preimage in $H^{1}(k, G)$ of the image of $X\left(\mathbb{A}_{k}\right)_{\bullet}$; this is completely analogous to the definition of a Selmer group in case $X$ is an abelian variety $A$, and $G=A[n]$ is the $n$-torsion subgroup of $A$.

Here are some basic properties. 
Lemma 5.3. (1) If $(\phi, \gamma):\left(Y^{\prime}, G^{\prime}\right) \rightarrow(Y, G)$ is a morphism in $\mathscr{C o v}(X)$, and if $P \in X\left(\mathbb{A}_{k}\right)$. survives $\left(Y^{\prime}, G^{\prime}\right)$, then $P$ also survives $(Y, G)$.

(2) If $\left(Y^{\prime}, G\right) \in \mathscr{C o v}\left(X^{\prime}\right)$ is the pull-back of $(Y, G) \in \mathscr{C o v}(X)$ under a morphism $\psi: X^{\prime} \rightarrow X$, then $P \in X^{\prime}\left(\mathbb{A}_{k}\right)$. survives $\left(Y^{\prime}, G\right)$ if and only if $\psi(P)$ survives $(Y, G)$.

(3) If $\left(Y_{1}, G_{1}\right),\left(Y_{2}, G_{2}\right) \in \mathscr{C o v}(X)$ have fiber product $(Y, G)$, then $P \in X\left(\mathbb{A}_{k}\right)$. survives $(Y, G)$ if and only if $P$ survives both $\left(Y_{1}, G_{1}\right)$ and $\left(Y_{2}, G_{2}\right)$.

(4) Let $X$ be over $K$, where $K / k$ is a finite extension, and let $(Y, G) \in \operatorname{Cov}(X)$ be an $X$-torsor. Then $P \in\left(R_{K / k} X\right)\left(\mathbb{A}_{k}\right)$. survives $\left(R_{K / k} Y, R_{K / k} G\right)$ if and only if its image in $X\left(\mathbb{A}_{K}\right)$. survives $(Y, G)$.

(5) If $(Y, G) \in \operatorname{Cov}(X)$ and $\xi \in H^{1}(k, G)$, then $P \in X\left(\mathbb{A}_{k}\right)$. survives $(Y, G)$ if and only if $P$ survives $\left(Y_{\xi}, G_{\xi}\right)$.

Proof. (1) By assumption, there are $\xi \in H^{1}\left(k, G^{\prime}\right)$ and $Q \in Y_{\xi}^{\prime}\left(\mathbb{A}_{k}\right)$. such that $\pi_{\xi}^{\prime}(Q)=P$. We have the morphism $\phi_{\xi}: Y_{\xi}^{\prime} \rightarrow Y_{\gamma_{*} \xi}$ over $X$; hence $\pi_{\gamma_{*} \xi}\left(\phi_{\xi}(Q)\right)=$ $\pi_{\xi}^{\prime}(Q)=P$, whence $P$ survives $(Y, G)$.

(2) Assume that $P$ survives $\left(Y^{\prime}, G\right)$. There are $\xi \in H^{1}(k, G)$ and $Q \in Y_{\xi}^{\prime}\left(\mathbb{A}_{k}\right)$. such that $\pi_{\xi}^{\prime}(Q)=P$. There is a morphism $\Psi_{\xi}: Y_{\xi}^{\prime} \rightarrow Y_{\xi}$ over $\psi$, and hence we have that $\pi_{\xi}\left(\Psi_{\xi}(Q)\right)=\psi(P)$, so $\psi(P)$ survives $(Y, G)$. Conversely, assume that $\psi(P)$ survives $(Y, G)$. Then there are $\xi \in H^{1}(k, G)$ and $Q \in Y_{\xi}\left(\mathbb{A}_{k}\right)$. such that $\pi_{\xi}(Q)=\psi(P)$. The twist $\left(Y_{\xi}^{\prime}, G_{\xi}\right)$ is the pull-back of $\left(Y_{\xi}, G_{\xi}\right)$ under $\psi$; in particular, $Y_{\xi}^{\prime}=Y_{\xi} \times{ }_{X} X^{\prime}$, and so there is $Q^{\prime} \in Y_{\xi}^{\prime}\left(\mathbb{A}_{k}\right)$ • mapping to $Q$ in $Y_{\xi}$ and to $P$ in $X^{\prime}$. Hence $P$ survives $\left(Y^{\prime}, G\right)$.

(3) We have obvious morphisms $(Y, G) \rightarrow\left(Y_{i}, G_{i}\right)$. So by part (1), if $P$ survives $(Y, G)$, then it also survives $\left(Y_{1}, G_{1}\right)$ and $\left(Y_{2}, G_{2}\right)$. Now assume that $P$ survives both $\left(Y_{1}, G_{1}\right)$ and $\left(Y_{2}, G_{2}\right)$. Then there are $\xi_{1} \in H^{1}\left(k, G_{1}\right)$ and $\xi_{2} \in H^{1}\left(k, G_{2}\right)$ and points $Q_{1} \in Y_{1, \xi_{1}}\left(\mathbb{A}_{k}\right)_{\bullet}, Q_{2} \in Y_{2, \xi_{2}}\left(\mathbb{A}_{k}\right)$. such that $\pi_{1, \xi_{1}}\left(Q_{1}\right)=P$ and $\pi_{2, \xi_{2}}\left(Q_{2}\right)=$ $P$. Consider $\xi=\left(\xi_{1}, \xi_{2}\right) \in H^{1}(k, G)=H^{1}\left(k, G_{1}\right) \times H^{1}\left(k, G_{2}\right)$. We have that $Y_{\xi}=Y_{1, \xi_{1}} \times{ }_{X} Y_{2, \xi_{2}}$; hence there is $Q \in Y_{\xi}\left(\mathbb{A}_{k}\right)$. mapping to $Q_{1}$ and $Q_{2}$ under the canonical maps $Y_{\xi} \rightarrow Y_{i, \xi_{i}}(i=1,2)$, and to $P$ under $\pi_{\xi}: Y_{\xi} \rightarrow X$. Hence $P$ survives $(Y, G)$.

(4) We have $H^{1}\left(k, R_{K / k} G\right)=H^{1}(K, G)$, and the corresponding twists are compatible. For any $\xi$ in this set, we have $R_{K / k} Y_{\xi}=\left(R_{K / k} Y\right)_{\xi}$, and the adelic points $\left(R_{K / k} Y_{\xi}\right)\left(\mathbb{A}_{k}\right)$. and $Y_{\xi}\left(\mathbb{A}_{K}\right)$ • are identified. The claim follows.

(5) This comes from the fact that every twist of $(Y, G)$ is also a twist of $\left(Y_{\xi}, G_{\xi}\right)$ and vice versa. 
By the Descent Theorem 5.1, it is clear that (the image in $X\left(\mathbb{A}_{k}\right) \bullet$ of) a rational point $P \in X(k)$ survives every torsor. Therefore it makes sense to study the set of adelic points that survive every torsor (or a suitable subclass of torsors) in order to obtain information on the location of the rational points within the adelic points. Note that the set of points in $X\left(\mathbb{A}_{k}\right)$. surviving a given torsor is closed - it is a finite union of images of compact sets $Y_{\xi}\left(\mathbb{A}_{k}\right)$. under continuous maps.

We are led to the following definitions.

Definition 5.4. Let $X$ be a smooth projective variety over $k$.

(1) $X\left(\mathbb{A}_{k}\right)_{\bullet}^{\mathrm{f}-\operatorname{cov}}=\left\{P \in X\left(\mathbb{A}_{k}\right)_{\bullet}: P\right.$ survives all $\left.(Y, G) \in \mathscr{C o v}(X)\right\}$.

(2) $X\left(\mathbb{A}_{k}\right)_{\bullet}^{\mathrm{f}-\text { sol }}=\left\{P \in X\left(\mathbb{A}_{k}\right)_{\bullet}: P\right.$ survives all $\left.(Y, G) \in \mathscr{Y o l}(X)\right\}$.

(3) $X\left(\mathbb{A}_{k}\right)_{\bullet}^{\mathrm{f}-\mathrm{ab}}=\left\{P \in X\left(\mathbb{A}_{k}\right)_{\bullet}: P\right.$ survives all $\left.(Y, G) \in \mathscr{A} b(X)\right\}$.

(The "f" in the superscripts stands for "finite", since we are dealing with torsors under finite group schemes only.)

By the remark made before the definition above, we have

$$
X(k) \subset \overline{X(k)} \subset X\left(\mathbb{A}_{k}\right)_{\bullet}^{\mathrm{f}-\operatorname{cov}} \subset X\left(\mathbb{A}_{k}\right)_{\bullet}^{\mathrm{f}-\mathrm{sol}} \subset X\left(\mathbb{A}_{k}\right)_{\bullet}^{\mathrm{f}-\mathrm{ab}} \subset X\left(\mathbb{A}_{k}\right)_{\bullet} .
$$

Here, $\overline{X(k)}$ is the topological closure of $X(k)$ in $X\left(\mathbb{A}_{k}\right)_{\text {. }}$.

Recall the "evaluation map" for $P \in X\left(\mathbb{A}_{k}\right) \bullet$ and $G$ a finite étale $k$-group scheme,

$$
\operatorname{ev}_{P, G}: H_{\text {êt }}^{1}(X, G) \longrightarrow \prod_{v} H^{1}\left(k_{v}, G\right)
$$

(the set on the left can be considered as the set of isomorphism classes of $X$-torsors under $G$ ) and the restriction map

$$
\operatorname{res}_{G}: H^{1}(k, G) \longrightarrow \prod_{v} H^{1}\left(k_{v}, G\right)
$$

In these terms, we have

$$
X\left(\mathbb{A}_{k}\right)_{\bullet}^{\mathrm{f}-\operatorname{cov}}=\bigcap_{G}\left\{P \in X\left(\mathbb{A}_{k}\right)_{\bullet}: \operatorname{im}\left(\operatorname{ev}_{P, G}\right) \subset \operatorname{im}\left(\operatorname{res}_{G}\right)\right\},
$$

where $G$ runs through all finite étale $k$-group schemes. We obtain $X\left(\mathbb{A}_{k}\right)_{\bullet}^{\mathrm{f} \text {-sol }}$ and $X\left(\mathbb{A}_{k}\right)_{\bullet}^{\text {f-ab }}$ in a similar way, by restricting $G$ to solvable or abelian group schemes.

In the definition above, we can restrict to $(Y, G)$ with $Y$ connected (over $k)$ if $X$ is connected: if we have $(Y, G)$ with $Y$ not connected, then let $Y_{0}$ by a connected component of $Y$, and let $G_{0} \subset G$ be the stabilizer of this component. Then $\left(Y_{0}, G_{0}\right)$ is again a torsor of the same kind as $(Y, G)$, and we have a morphism $\left(Y_{0}, G_{0}\right) \rightarrow(Y, G)$. Hence, by Lemma 5.3, (1), if $P$ survives $\left(Y_{0}, G_{0}\right)$, then it also survives $(Y, G)$. 
However, we cannot restrict to geometrically connected torsors when $X$ is geometrically connected. The reason is that there can be obstructions coming from the fact that a suitable geometrically connected torsor does not exist.

Lemma 5.5. Assume that $X$ is geometrically connected. If there is a torsor $(Y, G) \in \mathscr{C o v}(X)$ such that $Y$ and all twists $Y_{\xi}$ are $k$-connected, but not geometrically connected, then $X\left(\mathbb{A}_{k}\right)_{\bullet}^{\mathrm{f}-\mathrm{cov}}=\varnothing$. The analogous statement holds for the solvable and abelian versions.

Proof. If $Y_{\xi}$ is connected, but not geometrically connected, then $Y_{\xi}\left(\mathbb{A}_{k}\right)_{\bullet}=\varnothing$ (this is because the finite scheme $\pi_{0}\left(Y_{\xi}\right)$ is irreducible and therefore satisfies the Hasse Principle, compare the proof of Proposition 5.12). Hence no point in $X\left(\mathbb{A}_{k}\right)$. survives $(Y, G)$.

Let us briefly discuss how this relates to the geometric fundamental group of $X$ over $\bar{k}$, assuming $X$ to be geometrically connected. In the following, we write $\bar{X}=X \times{ }_{k} \bar{k}$ and so forth, for the base-change of $X$ to a variety over $\bar{k}$. Every torsor $(Y, G) \in \mathscr{C o v}(X)(\mathscr{Y}$ ol $(X)$ or $\mathscr{A} b(X)$, respectively) gives rise to a covering $\bar{Y} \rightarrow \bar{X}$ that is Galois with (solvable or abelian) Galois group $G(\bar{k})$. The stabilizer $\Gamma$ of a connected component of $\bar{Y}$ is then a finite quotient of the geometric fundamental group $\pi_{1}(\bar{X})$. If we fix an embedding $k \rightarrow \mathbb{C}$, then $\pi_{1}(\bar{X})$ is the profinite completion of the topological fundamental group $\pi_{1}(X(\mathbb{C}))$, so $\Gamma$ is also a finite quotient of $\pi_{1}(X(\mathbb{C}))$. If $\Gamma$ is trivial, then $\pi_{0}(Y)$ is a $k$-torsor under $G$, and $(Y, G)$ is the pull-back of $\left(\pi_{0}(Y), G\right)$ under the structure morphism $X \rightarrow$ Spec $k$. We call such a torsor trivial. Note that all points in $X\left(\mathbb{A}_{k}\right)$. survive a trivial torsor (since their image in $(\operatorname{Spec} k)\left(\mathbb{A}_{k}\right)_{\bullet}=(\operatorname{Spec} k)(k)=\{\mathrm{pt}\}$ survives everything $)$; therefore trivial torsors do not give information.

Conversely, given a finite quotient $\Gamma$ of $\pi_{1}(\bar{X})$ or of $\pi_{1}(X(\mathbb{C}))$, there is a corresponding covering $\bar{Y} \rightarrow \bar{X}$ that will be defined over some finite extension $K$ of $k$. Let $\pi: Y \rightarrow X_{K}$ be the covering over $K$; it is a torsor under a $K$-group scheme $G$ such that $G(\bar{k})=\Gamma$. We now construct a torsor $\left(Z, R_{K / k} G\right) \in \operatorname{Cov}(X)$ that over $K$ factors through $\pi$. By restriction of scalars, we obtain $\left(R_{K / k} Y, R_{K / k} G\right) \in$ $\operatorname{Cov}\left(R_{K / k} X_{K}\right)$. We pull back via the canonical morphism $X \rightarrow R_{K / k} X_{K}$ to obtain $\left(Z, R_{K / k} G\right) \in \operatorname{Cov}(X)$. Over $K$, we have the following diagram

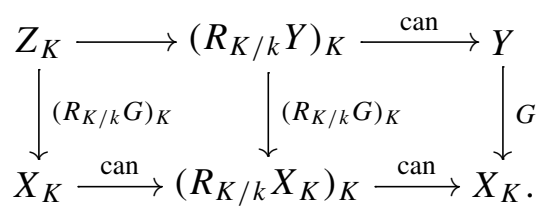

(Here the right hand horizontal maps come from the identity morphism $W \rightarrow W$ of a $K$-variety $W$, under the identification of $\operatorname{Mor}_{k}\left(V, R_{K / k} W\right)$ with $\operatorname{Mor}_{K}\left(V_{K}, W\right)$, taking $V=R_{K / k} W$; for $W=Y$ and $W=X_{K}$, respectively.) The composition 
of the lower horizontal maps is the identity morphism; hence $\left(Z_{K},\left(R_{K / k} G\right)_{K}\right) \in$ $\operatorname{Cov}\left(X_{K}\right)$ maps to $(Y, G)$. Note that the torsor we construct is in $\operatorname{Sol}(X)$ (respectively, $A b(X))$ when $\Gamma$ is solvable (respectively, abelian).

Lemma 5.6. Let $X$ be geometrically connected, $(Y, G),\left(Y^{\prime}, G^{\prime}\right) \in \mathscr{C o v}(X)$ such that $Y$ is geometrically connected and such that $(\bar{Y}, \bar{G})$ maps to $\left(\bar{Y}^{\prime}, \bar{G}^{\prime}\right)$ as torsors of $\bar{X}$. Then there is a twist $\left(Y_{\xi}^{\prime}, G_{\xi}^{\prime}\right)$ of $\left(Y^{\prime}, G^{\prime}\right)$ such that $(Y, G)$ maps to $\left(Y_{\xi}^{\prime}, G_{\xi}^{\prime}\right)$. Proof. Let $(\phi, \gamma):(\bar{Y}, \bar{G}) \rightarrow\left(\bar{Y}^{\prime}, \bar{G}^{\prime}\right)$ be the given morphism. Note that by assumption, the covering maps $\pi: Y \rightarrow X$ and $\pi^{\prime}: Y^{\prime} \rightarrow X$ are defined over $k$. For $\sigma \in \mathscr{G}_{k}$, this implies that $\left({ }^{\sigma} \phi,{ }^{\sigma} \gamma\right)$ is also a morphism $(\bar{Y}, \bar{G}) \rightarrow\left(\bar{Y}^{\prime}, \bar{G}^{\prime}\right)$. We can then consider the composite morphism

$$
\bar{Y} \stackrel{\left(\phi,{ }^{\sigma} \phi\right)}{\longrightarrow} \bar{Y}^{\prime} \times \bar{X} \bar{Y}^{\prime} \stackrel{\cong}{\longrightarrow} \bar{Y}^{\prime} \times \bar{G}^{\prime} \stackrel{\mathrm{pr}_{2}}{\longrightarrow} \bar{G}^{\prime} .
$$

Since $\bar{Y}$ is connected and $\bar{G}^{\prime}$ is discrete, this morphism must be constant. Let $\xi_{\sigma} \in G^{\prime}(\bar{k})$ be its image. It can then be checked that $\xi=\left(\xi_{\sigma}\right)_{\sigma \in \varphi_{k}}$ is a $G^{\prime}$-valued cocycle and that after twisting $\left(Y^{\prime}, G^{\prime}\right)$ by $\xi$, the morphism $\phi$ becomes defined over $k$; since $\gamma$ is uniquely determined by $\phi$, the same is true for $\gamma$.

We still assume $X$ to be geometrically connected. Let us call a family of torsors $\left(Y_{i}, G_{i}\right) \in \operatorname{Cov}(X)\left(\mathscr{S o l}(X)\right.$ or $\mathscr{A} b(X)$, respectively) with $Y_{i}$ geometrically connected a cofinal family of coverings of $X$ (respectively, of solvable or abelian coverings of $X)$ if for every (respectively, every solvable or abelian) connected $(\bar{Y}, \bar{G}) \in \mathscr{C o v}(\bar{X})$ (respectively, $\operatorname{Sol}(\bar{X})$ or $\mathscr{A} b(\bar{X}))$, there is a torsor $\left(Y_{i}, G_{i}\right)$ such that $\left(\bar{Y}_{i}, \bar{G}_{i}\right)$ maps to $(\bar{Y}, \bar{G})$. We then have the following.

Lemma 5.7. Let $X$ be geometrically connected.

(1) If $X\left(\mathbb{A}_{k}\right)^{\mathrm{f}-\mathrm{cov}} \neq \varnothing$, then there is a cofinal family of coverings of $X$. A similar statement holds for $X\left(\mathbb{A}_{k}\right)_{\bullet}^{\mathrm{f}-\mathrm{sol}}$ and solvable coverings, and for $X\left(\mathbb{A}_{k}\right)_{\bullet}^{\mathrm{f}-\mathrm{ab}}$ and abelian coverings.

(2) If $\left(Y_{i}, G_{i}\right)_{i}$ is a cofinal family of coverings of $X$, then $P \in X\left(\mathbb{A}_{k}\right)_{\bullet}$ is in $X\left(\mathbb{A}_{k}\right)_{\cdot}^{\mathrm{f}-\mathrm{cov}}$ if and only if $P$ survives every $\left(Y_{i}, G_{i}\right)$. Similarly for the solvable and abelian variants.

Proof.

(1) Let $P \in X\left(\mathbb{A}_{k}\right)_{\bullet}^{\mathrm{f}-\mathrm{cov}}$, and let $\bar{Y} \rightarrow \bar{X}$ be a finite étale Galois covering with Galois group $\Gamma$. Then by the discussion before Lemma 5.6, there is a torsor $(Z, G) \in \mathscr{C o v}(X)$, which we can assume to be $k$-connected, such that $(\bar{Z}, \bar{G})$ maps to $(\bar{Y}, \Gamma)$. Without loss of generality (after perhaps twisting $(Z, G)$ ), we can assume that $(Z, G)$ lifts $P$. This implies that $Z$ is geometrically connected (compare Lemma 5.5). So if we take all torsors $(Z, G)$ obtained in this way, we obtain a cofinal family of coverings of $X$. The proof in the solvable and abelian cases is analogous. 
(2) The "only if" part is clear. So assume that $P$ survives all $\left(Y_{i}, G_{i}\right)$, and let $(Z, \Gamma) \in \mathscr{C o v}(X)$ be arbitrary. Let $\bar{Z}_{0}$ be a connected component of $\bar{Z}$, and let $\bar{\Gamma}_{0}$ be the stabilizer of $\bar{Z}_{0}$. Then there is some $\left(Y_{i}, G_{i}\right)$ such that $\left(\bar{Y}_{i}, \bar{G}_{i}\right) \rightarrow$ $\left(\bar{Z}_{0}, \bar{\Gamma}_{0}\right) \rightarrow(\bar{Z}, \bar{\Gamma})$; hence by Lemma 5.6 , there is a twist $\left(Z_{\xi}, \Gamma_{\xi}\right)$ such that $\left(Y_{i}, G_{i}\right)$ maps to it. Since $P$ survives $\left(Y_{i}, G_{i}\right)$ by assumption, it also survives $\left(Z_{\xi}, \Gamma_{\xi}\right)$ and therefore $(Z, \Gamma)$, by Lemma 5.3. The proof in the solvable and abelian cases is again analogous.

Lemma 5.8. Let $X$ be geometrically connected.

(1) If $\pi_{1}(\bar{X})$ is trivial (that is, $X$ is simply connected), then $X\left(\mathbb{A}_{k}\right)_{\bullet}^{\mathrm{f}-\mathrm{cov}}=X\left(\mathbb{A}_{k}\right)_{\bullet}$.

(2) If the abelianization $\pi_{1}(\bar{X})^{\mathrm{ab}}$ is trivial, then $X\left(\mathbb{A}_{k}\right)_{\bullet}^{\mathrm{f}-\mathrm{ab}}=X\left(\mathbb{A}_{k}\right)$.

(3) If $\pi_{1}(\bar{X})$ is abelian (respectively, solvable), then $X\left(\mathbb{A}_{k}\right)_{\bullet}^{\mathrm{f}-\mathrm{cov}}=X\left(\mathbb{A}_{k}\right)_{\bullet}^{\mathrm{f}-\mathrm{ab}}(\mathrm{re}$ spectively, $\left.X\left(\mathbb{A}_{k}\right)_{\bullet}^{\mathrm{f}-\operatorname{cov}}=X\left(\mathbb{A}_{k}\right)_{\bullet}^{\mathrm{f}-\mathrm{sol}}\right)$.

Proof.

(1) In this case, all torsors are trivial and are therefore survived by all points in $X\left(\mathbb{A}_{k}\right)_{\text {. }}$.

(2) Here the same holds for all abelian torsors.

(3) We always have $X\left(\mathbb{A}_{k}\right)_{\bullet}^{\mathrm{f}-\operatorname{cov}} \subset X\left(\mathbb{A}_{k}\right)_{\bullet}^{\mathrm{f}-\text { ab }}$. So let $P \in X\left(\mathbb{A}_{k}\right)_{\bullet}^{\mathrm{f}-\mathrm{ab}}$; then by Lemma 5.7, (1), there is a cofinal family $\left(Y_{i}, G_{i}\right)$ of abelian coverings of $X$, and since $\pi_{1}(\bar{X})$ is abelian, this is also a cofinal family of coverings without restriction. By part (2) of the same lemma, it suffices to check that $P$ survives all $\left(Y_{i}, G_{i}\right)$, which we know to be true, in order to conclude that $P \in X\left(\mathbb{A}_{k}\right)^{\mathrm{f}-\text { cov }}$. Similarly for the solvable variant.

We now list some fairly elementary properties of the sets $X\left(\mathbb{A}_{k}\right)_{\bullet}^{\mathrm{f}-\mathrm{ab} / \mathrm{f}-\mathrm{sol} / \mathrm{f}-\mathrm{cov}}$.

Proposition 5.9. If $X^{\prime} \stackrel{\psi}{\rightarrow} X$ is a morphism, then $\psi\left(X^{\prime}\left(\mathbb{A}_{k}\right)_{\bullet}^{\mathrm{f}-\operatorname{cov}}\right) \subset X\left(\mathbb{A}_{k}\right)_{\bullet}^{\mathrm{f}-\operatorname{cov}}$. Similarly for the solvable and abelian variants.

Proof. Let $P \in X^{\prime}\left(\mathbb{A}_{k}\right)_{\bullet}^{\mathrm{f}-\text { cov }}$, and let $(Y, G) \in \mathscr{C o v}(X)$ be an $X$-torsor. By assumption, $P$ survives the pull-back $\left(Y^{\prime}, G\right)$ of $(Y, G)$ under $\psi$, so by Lemma 5.3, part (2), $\psi(P)$ survives $(Y, G)$. Since $(Y, G)$ is arbitrary, $\psi(P) \in X\left(\mathbb{A}_{k}\right)_{\bullet}^{\mathrm{f}-\mathrm{cov}}$. The same proof works for the solvable and abelian variants.

Lemma 5.10. Let $Z=\operatorname{Spec} k \amalg \operatorname{Spec} k=\left\{P_{1}, P_{2}\right\}$. Then

$$
\left\{P_{1}, P_{2}\right\}=Z(k)=Z\left(\mathbb{A}_{k}\right)^{\mathrm{f}-\mathrm{ab}} .
$$

Proof. Let $Q \in Z\left(\mathbb{A}_{k}\right)$. and assume that $Q \notin Z(k)$. We have to show that $Q \notin$ $Z\left(\mathbb{A}_{k}\right)_{\bullet}^{\mathrm{f}-\mathrm{ab}}$. By assumption, there are places $v$ and $w$ of $k$ such that $Q_{v}=P_{1}$ and 
$Q_{w}=P_{2}$. We will consider torsors under $G=\mathbb{Z} / 2 \mathbb{Z}$. Pick some $\alpha \in k^{\times}$such that $\alpha \notin\left(k_{v}^{\times}\right)^{2}$ and $\alpha \notin\left(k_{w}^{\times}\right)^{2}$. Let $Y=\operatorname{Spec} k(\sqrt{\alpha}) \amalg(\operatorname{Spec} k \amalg \operatorname{Spec} k)$; then $(Y, G) \in \mathscr{A} b(Z)$ in an obvious way. We want to show that no twist $\left(Y_{\xi}, G\right)$ for $\xi \in H^{1}(k, G)=k^{\times} /\left(k^{\times}\right)^{2}$ lifts $Q$. Such a twist is of one of the following forms:

$$
\begin{aligned}
& \left(Y_{\xi}, G\right)=\operatorname{Spec} k(\sqrt{\alpha}) \amalg(\operatorname{Spec} k \amalg \operatorname{Spec} k), \\
& \left(Y_{\xi}, G\right)=(\operatorname{Spec} k \amalg \operatorname{Spec} k) \amalg \operatorname{Spec} k(\sqrt{\alpha}), \\
& \left(Y_{\xi}, G\right)=\operatorname{Spec} k(\sqrt{\beta}) \amalg \operatorname{Spec} k(\sqrt{\gamma}),
\end{aligned}
$$

where in the last case, $\beta$ and $\gamma$ are independent in $k^{\times} /\left(k^{\times}\right)^{2}$. In the first two cases, $Q$ does not lift, since in the first case, the first component does not lift $Q_{v}$, and in the second case, the second component does not lift $Q_{w}$ (by our choice of $\alpha$ ). In the third case, there is a set of places of $k$ of density $1 / 4$ that are inert in both $k(\sqrt{\beta})$ and $k(\sqrt{\gamma})$, so $Y_{\xi}\left(\mathbb{A}_{k}\right)_{\bullet}=\varnothing$. In particular, $Q$ does not lift to any of these twists.

Proposition 5.11. If $X=X_{1} \amalg X_{2} \amalg \cdots \amalg X_{n}$ is a disjoint union, then

$$
X\left(\mathbb{A}_{k}\right)_{\bullet}^{\mathrm{f}-\mathrm{cov}}=\coprod_{j=1}^{n} X_{j}\left(\mathbb{A}_{k}\right)_{\bullet}^{\mathrm{f}-\operatorname{cov}},
$$

and similarly for the solvable and abelian variants.

Proof. It is sufficient to consider the case $n=2$. We have maps $X_{1} \rightarrow X$ and $X_{2} \rightarrow X$, so by Proposition 5.9, $X_{1}\left(\mathbb{A}_{k}\right)_{\bullet}^{\mathrm{f}-\operatorname{cov}} \amalg X_{2}\left(\mathbb{A}_{k}\right)_{\bullet}^{\mathrm{f}-\operatorname{cov}} \subset X\left(\mathbb{A}_{k}\right)_{\bullet}^{\mathrm{f}-\operatorname{cov}}$ (same for the solvable and abelian variants). For the reverse inclusion, consider the morphism $X \rightarrow$ Spec $k \amalg$ Spec $k=Z$ mapping $X_{1}$ to the first point and $X_{2}$ to the second point. If $Q \in X\left(\mathbb{A}_{k}\right)_{\bullet}^{\mathrm{f}-\mathrm{ab}}$, then its image is in $Z\left(\mathbb{A}_{k}\right)_{\bullet}^{\mathrm{f} \text {-ab }}=Z(k)$ (by Proposition 5.9 again and Lemma 5.10). This means that $Q \in X_{1}\left(\mathbb{A}_{k}\right)$ • $U X_{2}\left(\mathbb{A}_{k}\right)_{\bullet}$. The claim then follows easily.

Proposition 5.12. If $Z$ is a (reduced) finite scheme, then $Z\left(\mathbb{A}_{k}\right)_{\bullet}^{\mathrm{f}-\mathrm{ab}}=Z(k)$.

Proof. By Proposition 5.11, it suffices to prove this when $Z=\operatorname{Spec} K$ is connected. But in this case, it is known that $Z$ satisfies the Hasse Principle. On the other hand, if $Z(k) \neq \varnothing$, then $Z=$ Spec $k$ and $Z\left(\mathbb{A}_{k}\right)$. has just one point, so $Z(k)=Z\left(\mathbb{A}_{k}\right)_{\text {.. }}$.

(The statement that Spec $K$ as a $k$-scheme satisfies the Hasse Principle comes down to the following fact.

Fact. If a group $G$ acts transitively on a finite set $X$ such that every $g \in G$ fixes at least one element of $X$, then $\# X=1$.

To see this, let $n=\# X$ and assume (without loss of generality) that $G \subset S_{n}$. The stabilizer $G_{x}$ of $x \in X$ is a subgroup of index $n$ in $G$. By assumption, $G=\bigcup_{x \in X} G_{x}$, 


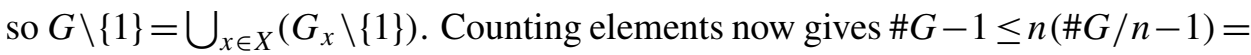
$\# G-n$, which implies $n=1$.)

Remark 5.13. Note that the Hasse Principle does not hold in general for finite schemes. A typical counterexample is given by the $\mathbb{Q}$-scheme

$\operatorname{Spec} \mathbb{Q}(\sqrt{13}) \amalg \operatorname{Spec} \mathbb{Q}(\sqrt{17}) \amalg \operatorname{Spec} \mathbb{Q}(\sqrt{13 \cdot 17})$.

Proposition 5.14. We have

$$
(X \times Y)\left(\mathbb{A}_{k}\right)_{\bullet}^{\mathrm{f}-\operatorname{cov}}=X\left(\mathbb{A}_{k}\right)_{\bullet}^{\mathrm{f}-\operatorname{cov}} \times Y\left(\mathbb{A}_{k}\right)_{\bullet}^{\mathrm{f}-\operatorname{cov}} .
$$

Similarly for the solvable and abelian variants.

Proof. Proposition 5.9 implies that

$$
(X \times Y)\left(\mathbb{A}_{k}\right)_{\bullet}^{\mathrm{f}-\operatorname{cov}} \subset X\left(\mathbb{A}_{k}\right)_{\bullet}^{\mathrm{f}-\operatorname{cov}} \times Y\left(\mathbb{A}_{k}\right)_{\bullet}^{\mathrm{f}-\operatorname{cov}}
$$

(and similarly for the solvable and abelian variants).

For the other direction, we can assume that $X$ and $Y$ are $k$-connected, compare Proposition 5.11. If $X$ (say) is not geometrically connected, then $X\left(\mathbb{A}_{k}\right)_{\bullet}=\varnothing$, and hence $(X \times Y)\left(\mathbb{A}_{k}\right)_{\bullet}=\varnothing$ as well, and the statement is trivially true. So we can assume that $X$ and $Y$ are geometrically connected.

We now use the fact that $\pi_{1}(\bar{X} \times \bar{Y})=\pi_{1}(\bar{X}) \times \pi_{1}(\bar{Y})$. Let $P \in X\left(\mathbb{A}_{k}\right)$ f-cov and $Q \in Y\left(\mathbb{A}_{k}\right)_{\bullet}^{\mathrm{f}-\text { cov }}$. By Lemma 5.7, (1), there are cofinal families of coverings $\left(V_{i}, G_{i}\right)$ of $X$ and $\left(W_{j}, H_{j}\right)$ of $Y$, which we can assume to lift $P$, respectively, $Q$. Then the products $\left(V_{i} \times W_{j}, G_{i} \times H_{j}\right)$ form a cofinal family of coverings of $X \times Y$, and it is clear that they lift $(P, Q)$. By Lemma 5.7, (2), this implies that $(P, Q) \in(X \times Y)\left(\mathbb{A}_{k}\right)^{\mathrm{f}-\operatorname{cov}}$.

The solvable and abelian variants are proved similarly, using the corresponding product property of the maximal abelian and solvable quotients of the geometric fundamental group.

Proposition 5.15. If $K / k$ is a finite extension and $X$ is a $K$-variety, then

$$
\left(R_{K / k} X\right)\left(\mathbb{A}_{k}\right)_{\bullet}^{\mathrm{f}-\operatorname{cov}}=X\left(\mathbb{A}_{K}\right)_{\bullet}^{\mathrm{f}-\operatorname{cov}}
$$

(under the canonical identification $\left.\left(R_{K / k} X\right)\left(\mathbb{A}_{k}\right)_{\bullet}=X\left(\mathbb{A}_{K}\right)_{\bullet}\right)$, and similarly for the solvable and abelian variants.

Proof. Let $P \in\left(R_{K / k} X\right)\left(\mathbb{A}_{k}\right)^{\mathrm{f}-\text { cov }}$, and let $(Y, G) \in \mathscr{C o v}(X)$. By assumption, $P$ survives $\left(R_{K / k} Y, R_{K / k} G\right) \in \mathscr{C o v}\left(R_{K / k} X\right)$, so by Lemma 5.3, part (4), $P$ also survives $(Y, G)$. Since $(Y, G)$ was arbitrary, $P \in X\left(\mathbb{A}_{K}\right)_{\bullet}^{\mathrm{f}-\text { cov }}$, so the left hand side is contained in the right hand side.

For the proof of the reverse inclusion, we can reduce to the case that $X$ is $K$-connected, by Proposition 5.11. If $X$ is $K$-connected, but not geometrically connected, then $\left(R_{K / k} X\right)\left(\mathbb{A}_{k}\right)_{\bullet}=X\left(\mathbb{A}_{K}\right)_{\bullet}=\varnothing$, and there is nothing to prove. So 
we can assume that $X$ is geometrically connected. Take $P \in X\left(\mathbb{A}_{K}\right)_{\bullet}^{\mathrm{f}-\mathrm{cov}}$. Then by Lemma 5.7, there is a cofinal family $\left(Y_{i}, G_{i}\right)$ of coverings of $X$. We show that $\left(R_{K / k} Y_{i}, R_{K / k} G_{i}\right)$ is then a cofinal family of coverings of $R_{K / k} X$. Indeed, it is known that $R_{K / k} X \cong \bar{X}^{[K: k]}$ (with the factors coming from the various embeddings of $K$ into $\bar{k}$ ), so $\pi_{1}\left(\overline{R_{K / k} X}\right) \cong \pi_{1}(\bar{X})^{[K: k]}$. This easily implies the claim. Now, viewing $P$ as an element of $\left(R_{K / k} X\right)\left(\mathbb{A}_{k}\right)_{\bullet}$, we see by Lemma 5.3 that $P$ survives every $\left(R_{K / k} Y_{i}, R_{K / k} G_{i}\right)$, and hence $P \in\left(R_{K / k} X\right)\left(\mathbb{A}_{k}\right)_{\bullet}^{\mathrm{f}-\mathrm{cov}}$.

The same proof works for the solvable and abelian variants.

Proposition 5.16. If $K / k$ is a finite extension, then

$$
X\left(\mathbb{A}_{k}\right)_{\bullet}^{\mathrm{f}-\operatorname{cov}} \subset X\left(\mathbb{A}_{k}\right) \bullet \cap\left(\mathbb{A}_{K}\right)_{\bullet}^{\mathrm{f}-\operatorname{cov}}
$$

and similarly for the solvable and abelian variants. Note that the intersection is to be interpreted as the pullback of $X\left(\mathbb{A}_{K}\right)_{\bullet}^{\mathrm{f}-\mathrm{cov}}$ under the canonical map $X\left(\mathbb{A}_{k}\right)_{\bullet} \rightarrow$ $X\left(\mathbb{A}_{K}\right)_{\bullet}$, which may not be injective at the infinite places.

Proof. We have a morphism $X \rightarrow R_{K / k} X_{K}$, inducing the canonical map

$$
X\left(\mathbb{A}_{k}\right) \bullet \longrightarrow\left(R_{K / k} X_{K}\right)\left(\mathbb{A}_{k}\right) \bullet=X\left(\mathbb{A}_{K}\right) \bullet
$$

The claim now follows from combining Propositions 5.9 and 5.15.

We also have an analogue of the Descent Theorem 5.1.

Proposition 5.17. Let $(Y, G) \in \mathscr{C o v}(X)$ be an $X$-torsor. Then

$$
X\left(\mathbb{A}_{k}\right)_{\bullet}^{\mathrm{f}-\operatorname{cov}}=\bigcup \pi_{\xi}\left(Y_{\xi}\left(\mathbb{A}_{k}\right)_{\bullet}^{\mathrm{f}-\operatorname{cov}}\right),
$$

where the union is extended over all twists $\left(Y_{\xi}, G_{\xi}\right)$ of $(Y, G)$, or equivalently, over the finite set of twists with points everywhere locally. A similar statement holds for the solvable variant, when $G$ is solvable.

Proof. Note first that by Proposition 5.9, the right hand side is a subset of the left hand side.

For the reverse inclusion, take $P \in X\left(\mathbb{A}_{k}\right)_{\bullet}^{\mathrm{f}-\mathrm{cov}}$. To ease notation, we will suppress the group schemes when denoting torsors in the following. Let $Y_{1}, \ldots, Y_{s} \in$ $\operatorname{Cov}(X)(\operatorname{or} \operatorname{Sol}(X))$ be the finitely many twists of $Y$ such that $P$ lifts.

Define $\tau(j) \subset\{1, \ldots, s\}$ to be the set of indices $i$ such that for every $X$-torsor $Z$ mapping to $Y_{j}$ (or an $X$-torsor $Z$ over $Y_{j}$ for short), there is a twist $Z_{\xi}$ that lifts $P$ and induces a twist of $Y_{j}$ that is isomorphic to $Y_{i}$. We make a number of claims about this function.

(i) $\tau(j)$ is nonempty. To see this, note first that for any given $Z$, the corresponding set (call it $\tau(Z)$ ) is nonempty, since by assumption $P$ must lift to some twist of $Z$, and this twist induces a twist of $Y_{j}$ to which $P$ also lifts, and hence this twist must be one of the $Y_{i}$. Second, if $Z$ maps to $Z^{\prime}$ (as $X$-torsors over $Y_{j}$ ), we have 
$\tau(Z) \subset \tau\left(Z^{\prime}\right)$. Third, for every pair of $X$-torsors $Z$ and $Z^{\prime}$ over $Y_{j}$, their relative fiber product $Z \times_{Y_{j}} Z^{\prime}$ maps to both of them. Taking these together, we see that $\tau(j)$ is a filtered intersection of nonempty subsets of a finite set and hence nonempty.

(ii) If $i \in \tau(j)$, then $\tau(i) \subset \tau(j)$. Let $h \in \tau(i)$, and let $Z$ be an $X$-torsor over $Y_{j}$. By definition of $\tau(j)$, there is a twist $Z_{\xi}$ of $Z$ lifting $P$ and inducing the twist $Y_{i}$ of $Y_{j}$. Now by definition of $\tau(i)$, there is a twist $\left(Z_{\xi}\right)_{\eta}$ of $Z_{\xi}$ lifting $P$ and inducing the twist $Y_{h}$ of $Y_{i}$. By transitivity of twists, this means that we have a twist of $Z$ lifting $P$ and inducing the twist $Y_{h}$ of $Y_{j}$. Since $Z$ was arbitrary, this shows that $h \in \tau(j)$.

(iii) For some $j$, we have $j \in \tau(j)$. Indeed, selecting for each $j$ some $\sigma(j) \in \tau(j)$ (this is possible by (i)), the map $\sigma$ will have a cycle: $\sigma^{m}(j)=j$ for some $m \geq 1$ and $j$. Then by (ii), it follows that $j \in \tau(j)$.

For this specific value of $j$, we have therefore proved that every $X$-torsor $Z$ over $Y_{j}$ has a twist that lifts $P$ and induces the trivial twist of $Y_{j}$. This means in particular that this twist is also a twist of $Z$ as a $Y_{j}$-torsor.

Now assume that $P$ does not lift to $Y_{j}\left(\mathbb{A}_{k}\right)_{\bullet}^{\mathrm{f}-\text { cov }}\left(\right.$ or $\left.Y_{j}\left(\mathbb{A}_{k}\right)_{\bullet}^{\mathrm{f}-\text { sol }}\right)$. Since the preimages of $P$ in $Y_{j}\left(\mathbb{A}_{k}\right)$. form a compact set and since surviving a torsor is a closed condition, we can find a $Y_{j}$-torsor $V$ that is not survived by any of the preimages of $P$. We can then find an $X$-torsor $Z$ mapping to $V$, staying in $\mathscr{Y} o l$ when working in that category. (Note that this step does not work for $\mathscr{A} b$, since extensions of abelian groups need not be abelian again.) But by what we have just proved, $Z$ has a twist as a $Y_{j}$-torsor that lifts a preimage of $P$, a contradiction. Hence our assumption that $P$ does not lift to $Y_{j}\left(\mathbb{A}_{k}\right)_{\bullet}^{\mathrm{f}-\operatorname{cov}}\left(\right.$ or $\left.Y_{j}\left(\mathbb{A}_{k}\right)_{\bullet}^{\mathrm{f}-\text { sol }}\right)$ must be false.

Remark 5.18. The analogous statement for $X\left(\mathbb{A}_{k}\right)_{\bullet}^{\mathrm{f}-\mathrm{ab}}$ and $G$ abelian is not true in general: it would follow that $X\left(\mathbb{A}_{k}\right)_{\bullet}^{\mathrm{f}-\mathrm{ab}}=X\left(\mathbb{A}_{k}\right)_{\bullet}^{\mathrm{f} \text {-sol }}$, but Skorobogatov (see [Skorobogatov 2001, § 8] or [Skorobogatov 1999]) has a celebrated example of a surface $X$ such that

$$
\varnothing=X\left(\mathbb{A}_{k}\right)_{\bullet}^{\mathrm{f}-\mathrm{sol}} \subsetneq X\left(\mathbb{A}_{k}\right)^{\mathrm{f}-\mathrm{ab}} .
$$

In fact, there is an abelian covering $\pi: Y \rightarrow X$ such that $\bigcup_{\xi} \pi_{\xi}\left(Y_{\xi}\left(\mathbb{A}_{k}\right)_{\bullet}^{\mathrm{f}-\mathrm{ab}}\right)=\varnothing$, which therefore gives a counterexample to the abelian version of the statement.

Skorobogatov shows that the "Brauer set" $X\left(\mathbb{A}_{k}\right)_{\bullet}^{\mathrm{Br}}$ is nonempty. In a later paper, Harari and Skorobogatov [2002, § 5.1] show that there exists an obstruction coming from a nilpotent, nonabelian covering (arising from an abelian covering of $Y$ ). The latter means that $X\left(\mathbb{A}_{k}\right)_{\bullet}^{\mathrm{f}-\mathrm{sol}}=\varnothing$, whereas the former implies that $X\left(\mathbb{A}_{k}\right)_{\bullet}^{\mathrm{f}-\mathrm{ab}} \neq \varnothing$, since $X\left(\mathbb{A}_{k}\right)_{\bullet}^{\mathrm{Br}} \subset X\left(\mathbb{A}_{k}\right)_{\bullet}^{\mathrm{f} \text {-ab }}$; see Section 7 below. The interest in this result comes from the fact that it is the first example known of a variety where there is no Brauer-Manin obstruction, yet there are no rational points. 


\section{Finite descent conditions and rational points}

The ultimate goal behind considering the sets cut out in the adelic points by the various covering conditions is to obtain information on the rational points. There is a three-by-three matrix of natural statements relating these sets; see the diagram below. Here, $\overline{X(k)}$ is the topological closure of $X(k)$ in $X\left(\mathbb{A}_{k}\right)$.

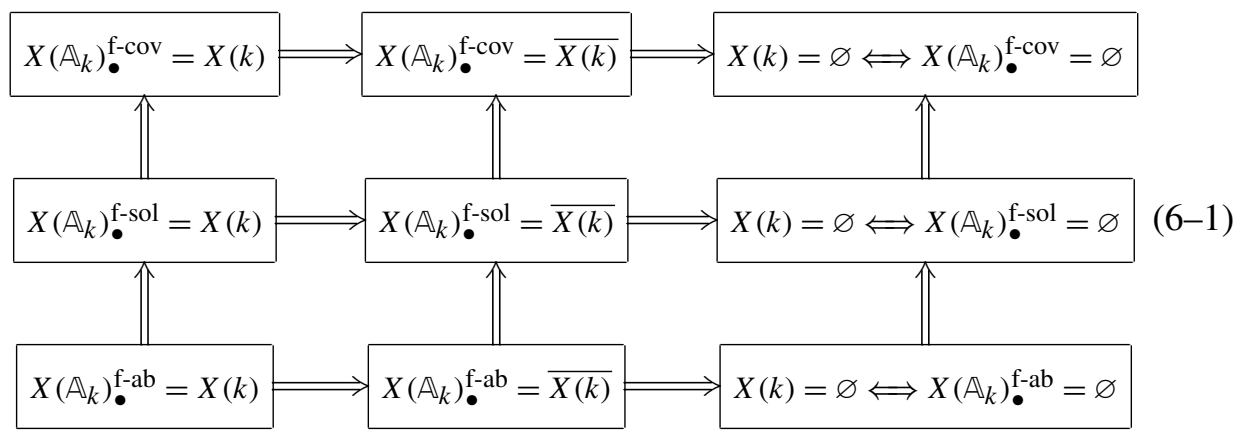

We have the implications shown. If $X(k)$ is finite, then we obviously have $X(k)=\overline{X(k)}$, and the corresponding statements in the left and middle columns are equivalent. In particular, this is the case when $X$ is a curve of genus at least 2 .

Let us discuss these statements. The ones in the middle column are perhaps the most natural ones, whereas the ones in the left column are better suited for proofs (as we will see below). The statements in the right column can be considered as variants of the Hasse Principle; in some sense they state that the Hasse Principle will eventually hold if one allows oneself to replace $X$ by finite étale coverings. Note that the weakest of the nine statements (the one in the upper right corner), if valid for a class of varieties, would imply that there is an effective procedure to decide whether there are $k$-rational points on a variety $X$ within that class or not: at least in principle, we can list all the $X$-torsors and for each torsor compute the finite set of twists with points everywhere locally. If this set is empty, we know that $X(k)=\varnothing$. In order to obtain the torsors, we can for example enumerate all finite extensions of the function field of $X$ (assuming that $X$ is geometrically connected, say) and check whether such an extension corresponds to an étale covering of $X$ that is a torsor under a finite group scheme. On the other hand, we can search for $k$-rational points on $X$ at the same time, and as soon as we find one such point, we know that $X(k) \neq \varnothing$. The statement $X(k)=\varnothing \Longleftrightarrow X\left(\mathbb{A}_{k}\right)_{\bullet}^{\mathrm{f}-\operatorname{cov}}=\varnothing$ guarantees that one of the two events must occur. (Note that $X\left(\mathbb{A}_{k}\right)_{\bullet}^{\mathrm{f}-\operatorname{cov}}$ can be written as a filtered intersection of compact subsets of $X\left(\mathbb{A}_{k}\right)_{\bullet}$, each coming from one specific torsor, so if $X\left(\mathbb{A}_{k}\right)_{\bullet}^{\mathrm{f}-\text { cov }}=\varnothing$, then already one of these conditions will provide an obstruction.) 
For $X$ of dimension at least two, none of these statements can be expected to hold in general. For example, a rational surface $X$ has trivial geometric fundamental group, and so $X\left(\mathbb{A}_{k}\right)_{\bullet}^{\mathrm{f}-\mathrm{cov}}=X\left(\mathbb{A}_{k}\right)_{\text {.. }}$. On the other hand, there are examples known of such surfaces that violate the Hasse principle, so we have $\varnothing=X(k) \subsetneq$ $X\left(\mathbb{A}_{k}\right)_{\bullet}^{\mathrm{f}-\mathrm{cov}}=X\left(\mathbb{A}_{k}\right)_{\text {. }}$. The first example (a smooth cubic surface) was given by Swinnerton-Dyer [1962]. There are also examples among smooth diagonal cubic surfaces, see [Cassels and Guy 1966], and in [Colliot-Thélène et al. 1980], an infinite family of rational surfaces violating the Hasse principle is given.

Let us give names to the properties in the left two columns in the diagram (6-1) above.

Definition 6.1. Let $X$ be a smooth projective $k$-variety. We call $X$

(1) good with respect to all coverings or simply good if $\overline{X(k)}=X\left(\mathbb{A}_{k}\right)_{\bullet}^{\mathrm{f}-\mathrm{cov}}$,

(2) good with respect to solvable coverings if $\overline{X(k)}=X\left(\mathbb{A}_{k}\right)_{\bullet}^{\mathrm{f}-\mathrm{sol}}$,

(3) good with respect to abelian coverings or very good if $\overline{X(k)}=X\left(\mathbb{A}_{k}\right)_{\bullet}^{\mathrm{f}-\mathrm{ab}}$,

(4) excellent with respect to all coverings if $X(k)=X\left(\mathbb{A}_{k}\right)_{\bullet}^{\mathrm{f}-\mathrm{cov}}$,

(5) excellent with respect to solvable coverings if $X(k)=X\left(\mathbb{A}_{k}\right)_{.}^{\mathrm{f}-\mathrm{sol}}$,

(6) excellent with respect to abelian coverings if $X(k)=X\left(\mathbb{A}_{k}\right)_{\bullet}^{\mathrm{f}-\mathrm{ab}}$.

Now let us look at curves in more detail. When $C$ is a curve of genus 0 , then it satisfies the Hasse Principle, so

$$
C\left(\mathbb{A}_{k}\right)_{\bullet}=\varnothing \Longleftrightarrow C(k)=\varnothing,
$$

and then all the intermediate sets are equal and empty. On the other hand, when $C(k) \neq \varnothing$, then $C \cong \mathbb{P}^{1}$, and $C(k)$ is dense in $C\left(\mathbb{A}_{k}\right)$., so

$$
\overline{C(k)}=C\left(\mathbb{A}_{k}\right)_{\bullet}^{\mathrm{f}-\mathrm{cov}}=C\left(\mathbb{A}_{k}\right)_{\bullet}^{\mathrm{f}-\mathrm{sol}}=C\left(\mathbb{A}_{k}\right)_{\bullet}^{\mathrm{f}-\mathrm{ab}}=C\left(\mathbb{A}_{k}\right)_{\bullet} .
$$

So curves of genus 0 are always very good.

Now consider the case of a genus 1 curve. If $A$ is an elliptic curve, or more generally, an abelian variety, then $\pi_{1}(\bar{A})$ is abelian, so by Lemma 5.8 we have

$$
A\left(\mathbb{A}_{k}\right)_{\bullet}^{\mathrm{f}-\mathrm{cov}}=A\left(\mathbb{A}_{k}\right)_{\bullet}^{\mathrm{f}-\mathrm{sol}}=A\left(\mathbb{A}_{k}\right)_{\bullet}^{\mathrm{f}-\mathrm{ab}} .
$$

Furthermore, among the abelian coverings, we can restrict to the multiplicationby- $n$ maps $A \stackrel{n}{\rightarrow} A$. (In the terminology used earlier, these coverings are a cofinal family.) This shows that

$$
A\left(\mathbb{A}_{k}\right)_{\bullet}^{\mathrm{f}-\mathrm{ab}}=\widehat{\operatorname{Sel}}(k, A) .
$$

Since the cokernel of the canonical map

$$
\overline{A(k)} \cong \widehat{A(k)} \longrightarrow \widehat{\operatorname{Sel}}(k, A)
$$


is the Tate module of $\amalg(k, A)$, we get the following.

Corollary 6.2. (1) A is very good if and only if $\amalg(k, A)_{\mathrm{div}}=0$.

(2) $A$ is excellent with respect to abelian coverings if and only if $A(k)$ is finite and $\amalg(k, A)_{\mathrm{div}}=0$.

See [Wang 1996] for a discussion of the situation when one works with $A\left(\mathbb{A}_{k}\right)$ instead of $A\left(\mathbb{A}_{k}\right)$. Note that Wang's discussion is in the context of the Brauer-Manin obstruction, which is closely related to the "finite abelian" obstruction considered here, as discussed in Section 7 below.

Corollary 6.3. If $A / \mathbb{Q}$ is a modular abelian variety of analytic rank zero, then $A$ is excellent with respect to abelian coverings. In particular, if $E / \mathbb{Q}$ is an elliptic curve of analytic rank zero, then $E$ is excellent with respect to abelian coverings.

Proof. In [Kolyvagin 1988; Kolyvagin and Logachëv 1989], it is proved that $A(\mathbb{Q})$ and $\amalg(\mathbb{Q}, A)$ are both finite. Corollary 6.2 then implies that $A\left(\mathbb{A}_{\mathbb{Q}}\right)_{\bullet}^{\mathrm{f}-\mathrm{ab}}=A(\mathbb{Q})$.

For elliptic curves $E / \mathbb{Q}$, Wiles [1995], Taylor and Wiles [1995], and Breuil, Conrad, Diamond and Taylor [Breuil et al. 2001] have proved that $E$ is modular, and so the first assertion applies.

Now let $X$ be a principal homogeneous space for the abelian variety $A$. If $X\left(\mathbb{A}_{k}\right)_{\bullet}=\varnothing$, then all statements in (6-1) are trivially true. So assume $X\left(\mathbb{A}_{k}\right) \bullet \neq \varnothing$, and let $\xi \in \amalg(k, A)$ denote the element corresponding to $X$. By Lemma 5.8, we have

$$
X\left(\mathbb{A}_{k}\right)_{\bullet}^{\mathrm{f}-\mathrm{cov}}=X\left(\mathbb{A}_{k}\right)_{\bullet}^{\mathrm{f}-\mathrm{sol}}=X\left(\mathbb{A}_{k}\right)_{\bullet}^{\mathrm{f}-\mathrm{ab}},
$$

and $X\left(\mathbb{A}_{k}\right)^{\mathrm{f}-\mathrm{ab}}=\varnothing$ if and only if $\xi \notin \amalg(k, A)_{\operatorname{div}}$. So for $\xi \neq 0, X$ is very good if and only if $\xi \notin \amalg(k, A)_{\text {div }}$ (since $X(k)=\varnothing$ in this case).

For curves $C$ of genus 2 or higher, we always have that $C(k)$ is finite, and so the statements in the left and middle columns in (6-1) are equivalent. In this case, we can characterize the set $C\left(\mathbb{A}_{k}\right)_{\bullet}^{\mathrm{f}-\mathrm{ab}}$ in a different way.

Theorem 6.4. Let $C$ be a smooth projective geometrically connected curve over $k$. Let $A=\mathrm{Alb}_{C}^{0}$ be its Albanese variety, and let $V=\mathrm{Alb}_{C}^{1}$ be the torsor under $A$ that parametrizes classes of zero-cycles of degree 1 on $C$. Then there is a canonical map $\phi: C \rightarrow V$, and we have

$$
C\left(\mathbb{A}_{k}\right)_{\bullet}^{\mathrm{f}-\mathrm{ab}}=\phi^{-1}\left(V\left(\mathbb{A}_{k}\right)_{\bullet}^{\mathrm{f}-\mathrm{ab}}\right) .
$$

Of course, since $C$ is a curve, $A$ is the same as the Jacobian variety $\mathrm{Jac}_{C}=\mathrm{Pic}_{C}^{0}$, and $V$ is its torsor $\operatorname{Pic}_{C}^{1}$, parametrizing divisor classes of degree 1 on $C$.

Proof. We know by Proposition 5.9 that $\phi\left(C\left(\mathbb{A}_{k}\right)_{\bullet}^{\mathrm{f}-\mathrm{ab}}\right) \subset V\left(\mathbb{A}_{k}\right)_{\bullet}^{\mathrm{f}-\mathrm{ab}}$. It therefore suffices to prove that $\phi^{-1}\left(V\left(\mathbb{A}_{k}\right)_{\bullet}^{\mathrm{f}-\mathrm{ab}}\right) \subset C\left(\mathbb{A}_{k}\right)_{\bullet}^{\mathrm{f}-\mathrm{ab}}$. 
By [Serre 1988, $\S$ VI.2], all (connected) finite abelian unramified coverings of $\bar{C}=C \times \times_{k} \bar{k}$ are obtained through pull-back from isogenies into $\bar{V} \cong \bar{A}$. From this, we can deduce that the induced homomorphism $\phi^{*}: H_{\mathrm{ett}}^{1}(\bar{V}, \bar{G}) \rightarrow H_{\mathrm{ett}}^{1}(\bar{C}, \bar{G})$ is an isomorphism for all finite abelian $k$-group schemes $G$. Since the map $\phi$ is defined over $k$, we obtain an isomorphism as $k$-Galois modules. The spectral sequence associated to the composition of functors $H^{0}\left(k, H_{\mathrm{ett}}^{0}(\bar{V},-)\right)=H_{\mathrm{ett}}^{0}(V,-)$ (and similarly for $C$ ) gives a diagram with exact rows,

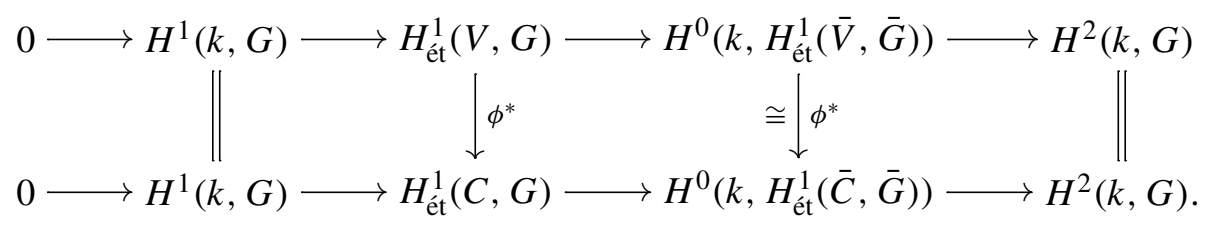

By the 5-lemma, $\phi^{*}: H_{\mathrm{et}}^{1}(V, G) \rightarrow H_{\mathrm{ett}}^{1}(C, G)$ is an isomorphism.

Let $P \in C\left(\mathbb{A}_{k}\right)_{\text {. such that }} \phi(P) \in V\left(\mathbb{A}_{k}\right)_{\bullet}^{\mathrm{f} \text {-ab }}$, and let $(Y, G) \in \mathscr{A} b(C)$. Then by the above, there is $(W, G) \in \mathscr{A} b(V)$ such that $Y$ is the pull-back of $W$. By assumption, $\phi(P)$ survives $(W, G)$; without loss of generality, $(W, G)$ already lifts $\phi(P)$. ( $G$ is abelian, hence equal to all its inner forms.) Then $(Y, G)$ lifts $P$, so $P$ survives $(Y, G)$. Since $(Y, G)$ was arbitrary, $P \in C\left(\mathbb{A}_{k}\right)_{\bullet}^{\mathrm{f}-\mathrm{ab}}$.

Remark 6.5. The result in the preceding theorem will hold more generally for smooth projective geometrically connected varieties $X$ instead of curves $C$, provided all finite étale abelian coverings of $\bar{X}$ can be obtained as pullbacks of isogenies into the Albanese variety of $X$. For this, it is necessary and sufficient that the (geometric) Néron-Severi group of $X$ is torsion-free; see [Serre 1988, VI.20].

For arbitrary varieties $X$, we can define a set $X\left(\mathbb{A}_{k}\right)_{\bullet}^{\text {Alb }}$ consisting of the adelic points on $X$ surviving all torsors that are pull-backs of $V$-torsors (where $V$ is the $k$-torsor under $A$ that receives a canonical map $\phi$ from $X$ ), and then the result above will hold in the form

$$
X\left(\mathbb{A}_{k}\right)_{\bullet}^{\mathrm{Alb}}=\phi^{-1}\left(V\left(\mathbb{A}_{k}\right)_{\bullet}^{\mathrm{f}-\mathrm{ab}}\right) .
$$

We trivially have $X\left(\mathbb{A}_{k}\right)_{\bullet}^{\mathrm{f}-\mathrm{ab}} \subset X\left(\mathbb{A}_{k}\right)_{\bullet}^{\text {Alb }}$.

In particular, we get that $X\left(\mathbb{A}_{k}\right)_{\bullet}^{\mathrm{Alb}}=X\left(\mathbb{A}_{k}\right)_{\bullet}$ if $X$ has trivial Albanese variety. For example, this is the case for all complete intersections of dimension at least 2 in some projective space. (By Exercise III.5.5 in [Hartshorne 1977], $H^{1}(\bar{X}, \mathcal{O})=0$ in this case, so the Picard variety and therefore also its dual $\operatorname{Alb}^{0}(X)$ are trivial.) If in addition $\mathrm{NS}_{X}$ is torsion-free, then $X\left(\mathbb{A}_{k}\right)_{\bullet}^{\mathrm{f}-\mathrm{ab}}=X\left(\mathbb{A}_{k}\right)_{\bullet}$ as well.

Corollary 6.6. Let $C$ be a smooth projective geometrically connected curve over $k$. Let $A$ be its Albanese (or Jacobian) variety, and let $V=\operatorname{Alb}_{C}^{1}=\operatorname{Pic}_{C}^{1}$ as above.

(1) If $C\left(\mathbb{A}_{k}\right)_{\bullet}=\varnothing$, then $C\left(\mathbb{A}_{k}\right)_{\bullet}^{\mathrm{f}-\mathrm{ab}}=C(k)=\varnothing$. 
(2) If $C\left(\mathbb{A}_{k}\right) \bullet \neq \varnothing$ and $V(k) \neq \varnothing$ (so that $C$ has a $k$-rational divisor class of degree 1), then there is a $k$-defined embedding $\phi: C \hookrightarrow A$, and we have

$$
C\left(\mathbb{A}_{k}\right)_{\bullet}^{\mathrm{f}-\mathrm{ab}}=\phi^{-1}(\widehat{\operatorname{Sel}}(k, A)) .
$$

If $\amalg(k, A)_{\mathrm{div}}=0$, we have

$$
C\left(\mathbb{A}_{k}\right)_{\bullet}^{\mathrm{f}-\mathrm{ab}}=\phi^{-1}(\overline{A(k)}) .
$$

(3) If $C\left(\mathbb{A}_{k}\right)_{\bullet} \neq \varnothing$ and $V(k)=\varnothing$, then, using the canonical map $\phi: C \rightarrow V$, we have

$$
C\left(\mathbb{A}_{k}\right)_{\bullet}^{\mathrm{f}-\mathrm{ab}}=\phi^{-1}\left(V\left(\mathbb{A}_{k}\right)_{\bullet}^{\mathrm{f}-\mathrm{ab}}\right) .
$$

Let $\xi \in \amalg(k, A)$ be the element corresponding to $V$. By assumption, $\xi \neq 0$. Then if $\xi \notin \amalg(k, A)_{\mathrm{div}}$ (and so in particular when $\left.\amalg(k, A)_{\mathrm{div}}=0\right)$, we have $C(k)=C\left(\mathbb{A}_{k}\right)_{\bullet}^{\mathrm{f}-\mathrm{ab}}=\varnothing$.

Similar statements are true for more general $X$ in place of $C$, with $X\left(\mathbb{A}_{k}\right)_{\bullet}^{\mathrm{Alb}}$ in place of $C\left(\mathbb{A}_{k}\right)^{\mathrm{f}-\mathrm{ab}}$.

Proof. This follows immediately from Theorem 6.4, taking into account the descriptions of $A\left(\mathbb{A}_{k}\right)_{\bullet}^{\mathrm{f}-\mathrm{ab}}$ and $V\left(\mathbb{A}_{k}\right)_{\bullet}^{\mathrm{f}-\mathrm{ab}}$ in Corollary 6.2 and the text following it.

Let $X$ be a smooth projective geometrically connected $k$-variety, let $A$ be its Albanese variety, and denote by $V$ the $k$-torsor under $A$ such that there is a canonical map $\phi: X \rightarrow V$. ( $V$ corresponds to the cocycle class of $\sigma \mapsto\left[P^{\sigma}-P\right] \in A(\bar{k})$ for any point $P \in X(\bar{k})$.) If $V(k) \neq \varnothing$, then $V$ is the trivial torsor, and there is an $n$-covering of $V$, that is, a $V$-torsor under $A[n]$. So the nonexistence of an $n$-covering of $V$ is an obstruction against rational points on $V$ and therefore on $X$.

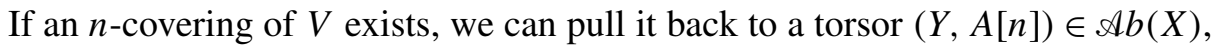
and we will say that a point $P \in X\left(\mathbb{A}_{k}\right)$. survives the $n$-covering of $X$ if it survives $(Y, A[n])$. If there is no $n$-covering, then by definition no point in $X\left(\mathbb{A}_{k}\right) \bullet$ survives the $n$-covering of $X$. If we denote the set of adelic points surviving the $n$-covering of $X$ by $X\left(\mathbb{A}_{k}\right)_{\bullet}^{n \text {-ab }}$, then we have

$$
X\left(\mathbb{A}_{k}\right)_{\bullet}^{\mathrm{Alb}}=\bigcap_{n \geq 1} X\left(\mathbb{A}_{k}\right)_{\bullet}^{n-\mathrm{ab}}
$$

In particular, for a curve $C$, we get

$$
C\left(\mathbb{A}_{k}\right)_{\bullet}^{\mathrm{f}-\mathrm{ab}}=\bigcap_{n \geq 1} C\left(\mathbb{A}_{k}\right)_{\bullet}^{n-\mathrm{ab}}
$$




\section{Relation with the Brauer-Manin obstruction}

In this section, we study the relationship between the finite covering obstructions introduced in Section 5 and the Brauer-Manin obstruction. This latter obstruction was introduced by Manin [1971] in 1970 in order to provide a unified framework to explain violations of the Hasse Principle.

The idea is as follows. Let $X$ be, as usual, a smooth projective geometrically connected $k$-variety. We then have the (cohomological) Brauer group

$$
\operatorname{Br}(X)=H_{\text {êt }}^{2}\left(X, \mathbb{G}_{m}\right) .
$$

If $K / k$ is any field extension and $P \in X(K)$ is a $K$-point of $X$, then the corresponding morphism Spec $K \rightarrow X$ induces a homomorphism $\phi_{P}: \operatorname{Br}(X) \rightarrow \operatorname{Br}(K)$. If $K=k_{v}$ is a completion of $k$, then there is a canonical injective homomorphism

$$
\operatorname{inv}_{v}: \operatorname{Br}\left(k_{v}\right) \hookrightarrow \mathbb{Q} / \mathbb{Z}
$$

(which is an isomorphism when $v$ is a finite place). In this way, we can set up a pairing

$$
X\left(\mathbb{A}_{k}\right) \bullet \times \operatorname{Br}(X) \longrightarrow \mathbb{Q} / \mathbb{Z}, \quad\left(\left(P_{v}\right), b\right) \longmapsto\left\langle\left(P_{v}\right), b\right\rangle_{B r}=\sum_{v} \operatorname{inv}_{v}\left(\phi_{P_{v}}(b)\right) .
$$

By a fundamental result of Class Field Theory, $k$-rational points on $X$ pair trivially with all elements of $\operatorname{Br}(X)$. This implies that

$$
\overline{X(k)} \subset X\left(\mathbb{A}_{k}\right)_{\bullet}^{\mathrm{Br}}=\left\{P \in X\left(\mathbb{A}_{k}\right)_{\bullet}:\langle P, b\rangle_{\mathrm{Br}}=0 \text { for all } b \in \operatorname{Br}(X)\right\} .
$$

The set $X\left(\mathbb{A}_{k}\right)^{\mathrm{Br}}$ is called the Brauer set of $X$. If it is empty, one says that there is a Brauer-Manin obstruction against rational points on $X$. More generally, if $B \subset \operatorname{Br}(X)$ is a subgroup (or subset), we can define $X\left(\mathbb{A}_{k}\right)_{\text {. }}^{B}$ in a similar way as the subset of points in $X\left(\mathbb{A}_{k}\right)$. that pair trivially with all $b \in B$.

The main result of this section is that for a curve $C$, we have

$$
C\left(\mathbb{A}_{k}\right)_{\bullet}^{\mathrm{Br}}=C\left(\mathbb{A}_{k}\right)_{\bullet}^{\mathrm{f}-\mathrm{ab}},
$$

see Corollary 7.3 below. This implies that all the results we have deduced or will deduce about finite abelian descent obstructions on curves also apply to the Brauer-Manin obstruction.

We first recall that the (algebraic) Brauer-Manin obstruction is at least as strong as the obstruction coming from finite abelian descent. For a more precise statement, see [Harari and Skorobogatov 2002, Thm. 4.9]. We define

$$
\operatorname{Br}_{1}(X)=\operatorname{ker}\left(\operatorname{Br}(X) \longrightarrow \operatorname{Br}\left(X \times_{k} \bar{k}\right)\right) \subset \operatorname{Br}(X)
$$

and set $X\left(\mathbb{A}_{k}\right)_{\bullet}^{\mathrm{Br}_{1}}=X\left(\mathbb{A}_{k}\right)_{\bullet}^{\mathrm{Br}(X)}$. 
Theorem 7.1. For any smooth projective geometrically connected variety $X$, we have

$$
X\left(\mathbb{A}_{k}\right)_{\bullet}^{\mathrm{Br}} \subset X\left(\mathbb{A}_{k}\right)_{\bullet}^{\mathrm{Br}} \subset X\left(\mathbb{A}_{k}\right)_{\bullet}^{\mathrm{f}-\mathrm{ab}} .
$$

Proof. The main theorem of descent theory of Colliot-Thélène and Sansuc [1987], as extended by Skorobogatov (see [Skorobogatov 1999] and [Skorobogatov 2001, Thm. 6.1.1]), states that $X\left(\mathbb{A}_{k}\right)_{\bullet}^{\mathrm{Br}_{1}}$ is equal to the set obtained from descent obstructions with respect to torsors under $k$-groups $G$ of multiplicative type, which includes all finite abelian $k$-groups. This proves the second inclusion. The first one follows from the definitions.

It is known that (see [Skorobogatov 2001, Cor. 2.3.9]; use that $H^{3}\left(k, \bar{k}^{\times}\right)=0$ )

$$
\frac{\mathrm{Br}_{1}(X)}{\mathrm{Br}_{0}(X)} \cong H^{1}\left(k, \operatorname{Pic}_{X}\right),
$$

where $\operatorname{Br}_{0}(X)$ denotes the image of $\operatorname{Br}(k)$ in $\operatorname{Br}(X)$. We also have the canonical map $H^{1}\left(k, \operatorname{Pic}_{X}^{0}\right) \rightarrow H^{1}\left(k, \operatorname{Pic}_{X}\right)$. Define $\operatorname{Br}_{1 / 2}(X)$ to be the subgroup of $\operatorname{Br}_{1}(X)$ that maps into the image of $H^{1}\left(k, \mathrm{Pic}_{X}^{0}\right)$ in $H^{1}\left(k, \mathrm{Pic}_{X}\right)$. (Manin [1971] calls it $\operatorname{Br}_{1}^{\prime}(X)$.) In addition, for $n \geq 1$, let $\operatorname{Br}_{1 / 2, n}(X)$ be the subgroup of $\operatorname{Br}_{1}(X)$ that maps into the image of $H^{1}\left(k, \operatorname{Pic}_{X}^{0}\right)[n]$. Then

$$
\operatorname{Br}_{1 / 2}(X)=\bigcup_{n \geq 1} \operatorname{Br}_{1 / 2, n}(X), \quad \text { and } \quad X\left(\mathbb{A}_{k}\right)_{\bullet}^{\mathrm{Br}_{1 / 2}}=\bigcap_{n \geq 1} X\left(\mathbb{A}_{k}\right)_{\bullet}^{\mathrm{Br}_{1 / 2, n}}
$$

Recall the definition of $X\left(\mathbb{A}_{k}\right)_{\bullet}^{\text {Alb }}$ from Remark 6.5 and the fact that

$$
X\left(\mathbb{A}_{k}\right)_{\bullet}^{\mathrm{f}-\mathrm{ab}} \subset X\left(\mathbb{A}_{k}\right)_{\bullet}^{\mathrm{Alb}}=\bigcap_{n \geq 1} X\left(\mathbb{A}_{k}\right)_{\bullet}^{n-\mathrm{ab}} .
$$

Theorem 7.2. Let $X$ be a smooth projective geometrically connected variety, and let $n \geq 1$. Then

$$
X\left(\mathbb{A}_{k}\right)_{\bullet}^{n-\mathrm{ab}} \subset X\left(\mathbb{A}_{k}\right)_{\bullet}^{\mathrm{Br}_{1 / 2, n}} .
$$

In particular,

$$
X\left(\mathbb{A}_{k}\right)_{\bullet}^{\mathrm{f}-\mathrm{ab}} \subset X\left(\mathbb{A}_{k}\right)_{\bullet}^{\mathrm{Alb}} \subset X\left(\mathbb{A}_{k}\right)_{\bullet}^{\mathrm{Br}_{1 / 2}} .
$$

Proof. Given the first statement, the second statement is clear.

The first statement follows from Theorem 7.5 below. However, since our proof of the inclusion given here is fairly simple, we include it.

So consider $P \in X\left(\mathbb{A}_{k}\right)_{0}^{n \text {-ab }}$ and $b \in \operatorname{Br}_{1 / 2, n}(X)$. We have to show that $\langle b, P\rangle_{\mathrm{Br}}$ vanishes, where $\langle\cdot, \cdot\rangle_{\mathrm{Br}}$ is the Brauer pairing between $X\left(\mathbb{A}_{k}\right)$. and $\operatorname{Br}(X)$.

Let $b^{\prime}$ be the image of $b$ in

$$
\mathrm{Br}_{1}(X) / \mathrm{Br}_{0}(X) \cong H^{1}\left(k, \operatorname{Pic}_{X}\right),
$$


and let $b^{\prime \prime} \in H^{1}\left(k, \operatorname{Pic}_{X}^{0}\right)[n]$ be an element mapping to $b^{\prime}$ (which exists because $\left.b \in \operatorname{Br}_{1 / 2, n}(X)\right)$.

Let $A$ be the Albanese variety of $X$, and let $V$ be the $k$-torsor under $A$ that has a canonical map $\phi: X \rightarrow V$. Then we have $\operatorname{Pic}_{X}^{0} \cong \operatorname{Pic}_{A}^{0} \cong \operatorname{Pic}_{V}^{0}$. Since $P \in X\left(\mathbb{A}_{k}\right)_{\bullet}^{n \text {-ab }} \stackrel{\phi}{\rightarrow} V\left(\mathbb{A}_{k}\right)_{\bullet}^{n \text {-ab }}$, the latter is nonempty, and hence $V$ admits a torsor of the form $(W, A[n])$.

Since $P$ maps into $V\left(\mathbb{A}_{k}\right)_{\bullet}^{n \text {-ab }}$, there is some twist of $(W, A[n])$ such that $\phi(P)$ lifts to it. Without loss of generality, $(W, A[n])$ is already this twist, so there is $Q^{\prime} \in W\left(\mathbb{A}_{k}\right)$. such that $\pi^{\prime}\left(Q^{\prime}\right)=\phi(P)$, where $\pi^{\prime}: W \rightarrow V$ is the covering map associated to $(W, A[n])$.

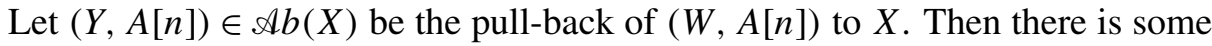
$Q \in Y\left(\mathbb{A}_{k}\right)$. such that $\pi(Q)=P$. Now the left hand diagram below induces the one on the right, where the rightmost vertical map is the multiplication by $n$.
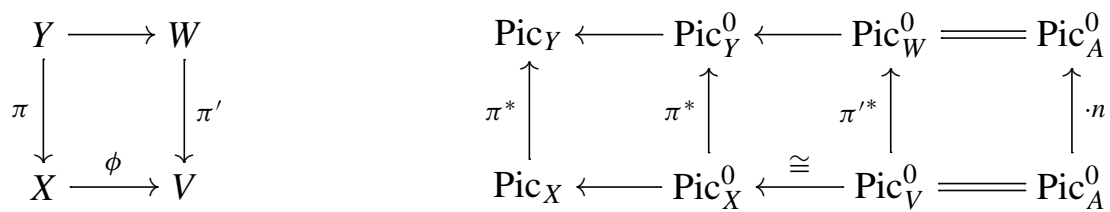

Chasing $b^{\prime \prime}$ around the diagram on the right, after applying $H^{1}(k,-)$ to it, we see that $\pi^{*}\left(b^{\prime}\right)=0$ in $\operatorname{Br}(Y) / \operatorname{Br}_{0}(Y)$. Finally, we have

$$
\langle b, P\rangle_{\mathrm{Br}}=\left\langle b^{\prime}, \pi(Q)\right\rangle_{\mathrm{Br}}=\left\langle\pi^{*}\left(b^{\prime}\right), Q\right\rangle_{\mathrm{Br}}=0 .
$$

So we have the chain of inclusions

$$
X\left(\mathbb{A}_{k}\right)_{\bullet}^{\mathrm{Br}} \subset X\left(\mathbb{A}_{k}\right)_{\bullet}^{\mathrm{Br}_{1}} \subset X\left(\mathbb{A}_{k}\right)_{\bullet}^{\mathrm{f}-\mathrm{ab}} \subset X\left(\mathbb{A}_{k}\right)_{\bullet}^{\mathrm{Alb}} \subset X\left(\mathbb{A}_{k}\right)_{\bullet}^{\mathrm{Br} r_{1 / 2}} .
$$

It is then natural to ask to what extent one might have equality in this chain of inclusions. We certainly get something when $\operatorname{Br}_{1 / 2}(X)$ already equals $\operatorname{Br}_{1}(X)$ or even $\operatorname{Br}(X)$.

Corollary 7.3. If $X$ is a smooth projective geometrically connected variety such that the canonical map $H^{1}\left(k, \operatorname{Pic}_{X}^{0}\right) \rightarrow H^{1}\left(k, \operatorname{Pic}_{X}\right)$ is surjective, then

$$
X\left(\mathbb{A}_{k}\right)_{\bullet}^{\mathrm{Br}_{1}}=X\left(\mathbb{A}_{k}\right)_{\bullet}^{\mathrm{f}-\mathrm{ab}}=X\left(\mathbb{A}_{k}\right)_{\bullet}^{\mathrm{Alb}} .
$$

In particular, if $C$ is a curve, then $C\left(\mathbb{A}_{k}\right)_{\bullet}^{\mathrm{Br}}=C\left(\mathbb{A}_{k}\right)_{\bullet}^{\mathrm{f}-\mathrm{ab}}$.

Proof. In this case, $\mathrm{Br}_{1 / 2}(X)=\mathrm{Br}_{1}(X)$, and so the result follows from the two preceding theorems.

When $X=C$ is a curve, then we know that $\operatorname{Br}\left(C \times_{k} \bar{k}\right)$ is trivial (Tsen's Theorem); also $H^{1}\left(k, \mathrm{Pic}_{C}^{0}\right)$ surjects onto $H^{1}\left(k, \mathrm{Pic}_{C}\right)$, since the Néron-Severi group 
of $C$ is $\mathbb{Z}$ with trivial Galois action, and $H^{1}(k, \mathbb{Z})=0$. Hence $\operatorname{Br}(C)=\operatorname{Br}_{1 / 2}(C)$, and the assertion follows.

The result of Corollary 7.3 means that we can replace $C\left(\mathbb{A}_{k}\right)_{\bullet}^{\mathrm{f}-a b}$ by $C\left(\mathbb{A}_{k}\right)_{\bullet}^{\mathrm{Br}}$ everywhere. For example, from Corollary 6.6, we obtain the following.

Corollary 7.4. Let $C$ be a smooth projective geometrically connected curve over $k$, and let $A$ be its Albanese (or Jacobian) variety. Assume that $\amalg(k, A)_{\operatorname{div}}=0$.

(1) If $C$ has a $k$-rational divisor class of degree 1 inducing a $k$-defined embedding $C \hookrightarrow A$, then

$$
C\left(\mathbb{A}_{k}\right)_{\bullet}^{\mathrm{Br}}=\phi^{-1}(\overline{A(k)}),
$$

where $\phi$ denotes the induced map $C\left(\mathbb{A}_{k}\right) \bullet \rightarrow A\left(\mathbb{A}_{k}\right)$.

(2) If $C$ has no $k$-rational divisor class of degree 1 , then $C\left(\mathbb{A}_{k}\right)_{\bullet}^{\mathrm{Br}}=\varnothing$.

These results can be found in Scharaschkin's thesis Scharaschkin [1999]. Our approach provides an alternative proof, and the more precise version in Corollary 6.6 shows how to extend the result to the case when the Shafarevich-Tate group of the Jacobian is not necessarily assumed to have trivial divisible subgroup.

We can strengthen Theorem 7.2.

Theorem 7.5. Let $X$ be a smooth projective geometrically connected variety. Then

$$
X\left(\mathbb{A}_{k}\right)_{\bullet}^{n-\mathrm{ab}}=X\left(\mathbb{A}_{k}\right)_{\bullet}^{\mathrm{Br}_{1 / 2, n}}
$$

for all $n \geq 1$. In particular,

$$
X\left(\mathbb{A}_{k}\right)_{\bullet}^{\mathrm{Alb}}=X\left(\mathbb{A}_{k}\right)_{\bullet}^{\mathrm{Br}_{1 / 2}} .
$$

Proof. This follows from the descent theory of Colliot-Thélène and Sansuc. Let $M=\operatorname{Pic}_{X}^{0}[n]$, and let $\lambda: M \rightarrow \operatorname{Pic}_{X}$ be the inclusion. Then the $n$-coverings of $X$ are exactly the torsors of type $\lambda$ in the language of the theory; compare for example [Skorobogatov 2001]. (Note that the dual of $M$ is $A[n]$, where $A$ is the Albanese variety of $X$.) We have $\mathrm{Br}_{\lambda}=\mathrm{Br}_{1 / 2, n}$, and the result then follows from Thm. 6.1.2,(a) in [Skorobogatov 2001].

Remark 7.6. Since $X\left(\mathbb{A}_{k}\right)_{\bullet}^{\mathrm{Br}_{1}} \subset X\left(\mathbb{A}_{k}\right)_{\bullet}^{\mathrm{f}-\mathrm{ab}} \subset X\left(\mathbb{A}_{k}\right)_{\bullet}^{\mathrm{Br}_{1 / 2}}$, it is natural to ask whether there might be a subgroup $B \subset \operatorname{Br}_{1}(X)$ such that $X\left(\mathbb{A}_{k}\right)_{\bullet}^{\mathrm{f}-\mathrm{ab}}=X\left(\mathbb{A}_{k}\right)_{\bullet}^{B}$. As Joost van Hamel pointed out to me, a natural candidate for $B$ is the subgroup mapping to the image of $H^{1}\left(k, \mathrm{Pic}_{X}^{\tau}\right)$ in $H^{1}\left(k, \mathrm{Pic}_{X}\right)$, where $\mathrm{Pic}_{X}^{\tau}$ is the saturation of $\mathrm{Pic}_{X}^{0}$ in $\mathrm{Pic}_{X}$, that is, the subgroup of elements mapping into the torsion subgroup of the Néron-Severi group $\mathrm{NS}_{X}$. It is tempting to denote this $B$ by $\mathrm{Br}_{2 / 3}$, but perhaps $\mathrm{Br}_{\tau}$ is the better choice. Note that $\mathrm{Br}_{\tau}=\mathrm{Br}_{1 / 2}$ when $\mathrm{NS}_{X}$ is torsion free, in which case we have $X\left(\mathbb{A}_{k}\right)_{\bullet}^{\mathrm{f}-\mathrm{ab}}=X\left(\mathbb{A}_{k}\right)_{\bullet}^{\mathrm{Alb}}=X\left(\mathbb{A}_{k}\right)_{\bullet}^{\mathrm{Br}_{1 / 2}}$. 
Corollary 7.7. If $C / k$ is a curve that has a rational divisor class of degree 1 , then

$$
C\left(\mathbb{A}_{k}\right)_{\bullet}^{n-\mathrm{ab}}=C\left(\mathbb{A}_{k}\right)_{\bullet}^{\mathrm{Br}[n]} .
$$

In words, the information coming from $n$-torsion in the Brauer group is exactly the information obtained by an $n$-descent on $C$.

Proof. Under the given assumptions, $H^{1}\left(k, \operatorname{Pic}_{C}^{0}\right)=H^{1}\left(k, \operatorname{Pic}_{C}\right)=\operatorname{Br}(C) / \operatorname{Br}(k)$, and $\operatorname{Br}(k)$ is a direct summand of $\operatorname{Br}(C)$. Therefore, the images of $\operatorname{Br}_{1 / 2, n}(C)$ and of $\operatorname{Br}(C)[n]$ in $\operatorname{Br}(C) / \mathrm{Br}_{0}(C)$ agree, and the claim follows.

Corollary 7.8. If $X$ is a smooth projective geometrically connected variety such that the Néron-Severi group of $X$ (over $\bar{k})$ is torsion-free, then there is a finite field extension $K / k$ such that

$$
X\left(\mathbb{A}_{K}\right)_{\bullet}^{\mathrm{Br}_{1}}=X\left(\mathbb{A}_{K}\right)_{\bullet}^{\mathrm{f}-\mathrm{ab}} .
$$

Proof. We have an exact sequence

$$
H^{1}\left(k, \operatorname{Pic}_{X}^{0}\right) \longrightarrow H^{1}\left(k, \operatorname{Pic}_{X}\right) \longrightarrow H^{1}\left(k, \mathrm{NS}_{X}\right) .
$$

Since $\mathrm{NS}_{X}$ is a finitely generated abelian group, the Galois action on it factors through a finite quotient $\operatorname{Gal}(K / k)$ of the absolute Galois group of $k$. Then

$$
H^{1}\left(K, \mathrm{NS}_{X}\right)=\operatorname{Hom}\left(G_{K}, \mathbb{Z}^{r}\right)=0,
$$

and the claim follows from Theorem 7.2.

Note that it is not true in general that $X\left(\mathbb{A}_{k}\right)_{\bullet}^{\mathrm{Br}_{1}}=X\left(\mathbb{A}_{k}\right)_{\bullet}^{\mathrm{f}-\mathrm{ab}}$ (even when the Néron-Severi group of $X$ over $\bar{k}$ is torsion-free). For example, a smooth cubic surface $X$ in $\mathbb{P}^{3}$ has $X\left(\mathbb{A}_{k}\right)_{\bullet}^{\mathrm{f}-\text { cov }}=X\left(\mathbb{A}_{k}\right) \bullet$ (since it has trivial geometric fundamental group), but may well have $X\left(\mathbb{A}_{k}\right)_{\bullet}^{\mathrm{Br}_{1}}=\varnothing$, even though there are points everywhere locally. See [Colliot-Thélène et al. 1987a], where the algebraic Brauer-Manin obstruction is computed for all smooth diagonal cubic surfaces

$$
X: a_{1} x_{1}^{3}+a_{2} x_{2}^{3}+a_{3} x_{3}^{3}+a_{4} x_{4}^{3}=0
$$

with integral coefficients $0<a_{i}<100$, thereby verifying that it is the only obstruction against rational points on $X$ (and thus providing convincing experimental evidence that this may be true for smooth cubic surfaces in general). This computation produces a list of 245 such surfaces with points everywhere locally, but no rational points, since $X\left(\mathbb{A}_{\mathbb{Q}}\right)_{\bullet}^{\mathrm{Br}_{1}}=\varnothing$.

It is perhaps worth mentioning that our condition that $H^{1}\left(k, \mathrm{Pic}_{X}^{0}\right)$ surjects onto $H^{1}\left(k, \operatorname{Pic}_{X}\right)$, which leads to the identification of the "algebraic Brauer-Manin obstruction" and the "finite abelian descent obstruction", is in some sense orthogonal to the situation studied (quite successfully) in [Colliot-Thélène and Sansuc 1987; Colliot-Thélène et al. 1980; 1987b], where it is assumed that $\mathrm{Pic}_{X}$ is torsion-free 
(and therefore $\mathrm{Pic}_{X}^{0}$ is trivial), and so there can be a Brauer-Manin obstruction only when our condition fails. There is then no finite abelian descent obstruction, and one has to look at torsors under tori instead.

In general, we have a diagram of inclusions:

$$
\begin{aligned}
& X(k) \subset \overline{X(k)} \subset X\left(\mathbb{A}_{k}\right)_{\bullet}^{\mathrm{Br}} \subset X\left(\mathbb{A}_{k}\right)_{\bullet}^{\mathrm{Br} r_{1}} \subset X\left(\mathbb{A}_{k}\right)_{\bullet}^{\mathrm{f}-\mathrm{ab}} \subset X\left(\mathbb{A}_{k}\right)_{\bullet}^{\mathrm{Br} r_{1 / 2}} \subset X\left(\mathbb{A}_{k}\right)_{\bullet} . \\
& \subset X\left(\mathbb{A}_{k}\right)_{\bullet}^{\mathrm{f}-\mathrm{cov}} \subset X\left(\mathbb{A}_{k}\right)_{\bullet}^{\mathrm{f}-\text { sol }} \subset
\end{aligned}
$$

We expect that every inclusion can be strict. We discuss them in turn.

(1) $X=\mathbb{P}^{1}$ has $X(k) \subsetneq \overline{X(k)}=X\left(\mathbb{A}_{k}\right)_{\bullet}$.

(2) Skorobogatov's famous example (see [Skorobogatov 1999; Harari and Skorobogatov 2002]) has $X\left(\mathbb{A}_{k}\right)_{\bullet}^{\mathrm{Br}} \neq \varnothing$, but $X\left(\mathbb{A}_{k}\right)_{\bullet}^{\mathrm{f}-\text { sol }}=\varnothing$, showing that $\overline{X(k)} \subsetneq$ $X\left(\mathbb{A}_{k}\right)_{\bullet}^{\mathrm{Br}}$ and $X\left(\mathbb{A}_{k}\right)_{\bullet}^{\mathrm{f}-\text { sol }} \subsetneq X\left(\mathbb{A}_{k}\right)_{\bullet}^{\mathrm{f} \text {-ab }}$ are both possible.

(3) As mentioned above, Colliot-Thélène et al. [1987a] have examples such that $X\left(\mathbb{A}_{k}\right)_{\bullet}^{\mathrm{Br}_{1}}=\varnothing$, but $X\left(\mathbb{A}_{k}\right)_{\bullet}^{\mathrm{f}-\text { cov }}=X\left(\mathbb{A}_{k}\right)_{\bullet}$. This shows that $\overline{X(k)} \subsetneq X\left(\mathbb{A}_{k}\right)_{\bullet}^{\mathrm{f}-\operatorname{cov}}$ and $X\left(\mathbb{A}_{k}\right)_{\bullet}^{\mathrm{Br}_{1}} \subsetneq X\left(\mathbb{A}_{k}\right)_{\bullet}^{\mathrm{f}-\mathrm{ab}}$ are both possible.

(4) [Harari 1996] has examples, where there is a "transcendental", but no "algebraic" Brauer-Manin obstruction, which means that $X\left(\mathbb{A}_{k}\right)_{\bullet}^{\mathrm{Br}}=\varnothing$, but $X\left(\mathbb{A}_{k}\right)_{\bullet}^{\mathrm{Br}} \neq \varnothing$. Hence we can have $X\left(\mathbb{A}_{k}\right)_{\bullet}^{\mathrm{Br}} \subsetneq X\left(\mathbb{A}_{k}\right)_{\bullet}^{\mathrm{Br}_{1}}$.

(5) If we take a finite nonabelian simple group for $\pi_{1}(\bar{X})$ in Cor. 6.1 in [Harari 2000], then the proof of this result shows that $X\left(\mathbb{A}_{k}\right)_{\bullet}^{\mathrm{f}-\mathrm{cov}} \subsetneq X\left(\mathbb{A}_{k}\right)_{\text {. }}$. On the other hand, $X\left(\mathbb{A}_{k}\right)_{\bullet}^{\mathrm{f}-\mathrm{sol}}=X\left(\mathbb{A}_{k}\right)_{\bullet}$, since there are only trivial torsors in $\mathscr{S o l}(X)$; compare Lemma 5.8.

(6) A construction using Enriques surfaces like that in [Harari and Skorobogatov 2005] should produce an example such that $X\left(\mathbb{A}_{k}\right)_{\bullet}^{\mathrm{Br}_{1 / 2}}=X\left(\mathbb{A}_{k}\right)_{\bullet}^{\mathrm{Alb}}=X\left(\mathbb{A}_{k}\right)_{\bullet}$, since the Albanese variety is trivial, but $X\left(\mathbb{A}_{k}\right)_{\bullet}^{\mathrm{f}-\mathrm{ab}} \subsetneq X\left(\mathbb{A}_{k}\right)_{\bullet}$, since there is a nontrivial abelian covering.

(7) Finally, in Section 8 below, we will see many examples of curves $X$ that have $X(k)=X\left(\mathbb{A}_{k}\right)_{\bullet}^{\mathrm{Br}_{1 / 2}} \subsetneq X\left(\mathbb{A}_{k}\right)$.

A new obstruction? For curves, we expect the interesting part of the diagram of inclusions above to collapse: $\overline{X(k)}=X\left(\mathbb{A}_{k}\right)_{\bullet}^{\mathrm{Br}_{1 / 2}}$; see the discussion in Section 9 below. For higher-dimensional varieties, this is far from true; see the discussion above. So one could consider a new obstruction obtained from a combination of the Brauer-Manin and the finite descent obstructions, as follows. Define

$$
X\left(\mathbb{A}_{k}\right)_{\bullet}^{\mathrm{f}-\operatorname{cov}, \mathrm{Br}}=\bigcap_{(Y, G) \in \mathscr{C o v}(X)} \bigcup_{\xi \in H^{1}(k, G)} \pi_{\xi}\left(Y_{\xi}\left(\mathbb{A}_{k}\right)_{\bullet}^{\mathrm{Br}}\right) .
$$


(This is similar in spirit to the "refinement of the Manin obstruction" introduced in [Skorobogatov 1999].)

It would be interesting to find out how strong this obstruction is and whether it is strictly weaker than the obstruction obtained from all torsors under (not necessarily finite or abelian) $k$-group schemes. Note that the latter is at least as strong as the Brauer-Manin obstruction by [Harari and Skorobogatov 2002, Thm. 4.10] (see also Prop. 5.3.4 in [Skorobogatov 2001]), at least if one assumes that all elements of $\operatorname{Br}(X)$ are represented by Azumaya algebras over $X$.

\section{Finite descent conditions on curves}

Let us now prove some general properties of the notions, introduced in Section 6 above, of being excellent with respect to all, solvable, or abelian coverings in the case of curves. In the following, $C, D$, and so forth, will be (smooth projective geometrically connected) curves over $k$. We will use $\iota$ to denote an embedding of $C$ into its Jacobian (if it exists). Also, if $C\left(\mathbb{A}_{k}\right)_{\bullet}^{\mathrm{Br}}=\varnothing$ (and therefore $C(k)=\varnothing$, too), we say that the absence of rational points is explained by the Brauer-Manin obstruction. Note that by Corollary 7.3, $C\left(\mathbb{A}_{k}\right)_{\bullet}^{\mathrm{Br}}=C\left(\mathbb{A}_{k}\right)_{\bullet}^{\mathrm{f}-\mathrm{ab}}$, which implies that the absence of rational points is explained by the Brauer-Manin obstruction when $C$ is excellent with respect to abelian coverings and $C(k)=\varnothing$. We will use this observation below without explicit mention.

Corollary 8.1. Let $C / k$ be a curve of genus at least 1 , with Jacobian J. Assume that $\amalg(k, J)_{\mathrm{div}}=0$ and that $J(k)$ is finite. Then $C$ is excellent with respect to abelian coverings. If $C(k)=\varnothing$, then the absence of rational points is explained by the Brauer-Manin obstruction.

Proof. By Corollary 6.6, under the assumption on $\amalg(k, J)$, either $C\left(\mathbb{A}_{k}\right)_{\bullet}^{\mathrm{f}-\mathrm{ab}}=\varnothing$, and there is nothing to prove, or else

$$
C\left(\mathbb{A}_{k}\right)_{\bullet}^{\mathrm{f}-\mathrm{ab}}=\iota^{-1}(\overline{J(k)})=\iota^{-1}(J(k))=C(k) .
$$

The following result shows that the statement we would like to have, namely that $C\left(\mathbb{A}_{k}\right)_{\bullet}^{\mathrm{f}-\mathrm{ab}}=C(k)$, holds for finite subschemes of a curve.

Theorem 8.2. Let $C / k$ be a curve of genus at least 1 , and let $Z \subset C$ be a finite subscheme. Then the image of $Z\left(\mathbb{A}_{k}\right)_{\bullet}$ in $C\left(\mathbb{A}_{k}\right)$ • meets $C\left(\mathbb{A}_{k}\right)_{\bullet}^{\mathrm{f}-\mathrm{ab}}$ in $Z(k)$. More generally, if $P \in C\left(\mathbb{A}_{k}\right)_{\bullet}^{\mathrm{f}-\mathrm{ab}}$ is such that $P_{v} \in Z\left(k_{v}\right)$ for a set of places $v$ of $k$ of density 1 , then $P \in Z(k)$.

Proof. Let $K / k$ be a finite extension such that $C$ has a rational divisor class of degree 1 over $K$. By Corollary 6.6, we have that

$$
C\left(\mathbb{A}_{K}\right)_{\bullet}^{\mathrm{f}-\mathrm{ab}}=\iota^{-1}(\widehat{\operatorname{Sel}}(K, J)),
$$


where $\iota: C\left(\mathbb{A}_{K}\right)_{\bullet} \rightarrow J\left(\mathbb{A}_{K}\right) \bullet$ is the map induced by an embedding $C \hookrightarrow J$ over $K$. Now we apply Theorem 3.11 to the image of $Z$ in $J$. We find that $\iota(P) \in \widehat{\operatorname{Sel}}(K, J)$ and so $\iota(P) \in \iota(Z(K))$. Since $\iota$ is injective (even at the infinite places!), we find that the image of $P$ in $C\left(\mathbb{A}_{K}\right)_{\bullet}$ is in (the image of) $Z(k)$. Now if $Z(k)$ is empty, this gives a contradiction and proves the claim in this case. Otherwise, $C(k) \supset Z(k)$ is nonempty, and we can take $K=k$ above, which gives the statement directly.

The following results show that the "excellence properties" behave nicely.

Proposition 8.3. Let $K / k$ be a finite extension, and let $C / k$ be a curve of genus at least 1 . If $C_{K}$ is excellent with respect to all, solvable, or abelian coverings, then so is $C$.

Proof. By Proposition 5.16, we have

$$
C(k) \subset C\left(\mathbb{A}_{k}\right)_{\bullet}^{\mathrm{f}-\operatorname{cov}} \subset C\left(\mathbb{A}_{k}\right)_{\bullet} \cap C\left(\mathbb{A}_{K}\right)_{\bullet}^{\mathrm{f}-\operatorname{cov}}=C\left(\mathbb{A}_{k}\right) \bullet \cap C(K)=C(k) .
$$

Similarly for $C\left(\mathbb{A}_{k}\right)_{0}^{\mathrm{f}-\text { sol }}$ and $C\left(\mathbb{A}_{k}\right)_{0}^{\mathrm{f}-\mathrm{ab}}$. Strictly speaking, this means that $C(k)$ and $C\left(\mathbb{A}_{k}\right)^{\mathrm{f}-\text { cov }}$ have the same image in $C\left(\mathbb{A}_{K}\right)_{\text {. }}$. Now, since $C(K)$ has to be finite in order to equal $C\left(\mathbb{A}_{K}\right)_{\bullet}^{\mathrm{f}-\mathrm{cov}}, C(k)$ is also finite, and we can apply Theorem 8.2 to $Z=C(k) \subset C$ and the set of finite places of $k$.

Proposition 8.4. Let $(D, G) \in \mathscr{C o v}(C)(\operatorname{or} \mathscr{Y}$ ol $(C))$. If all twists $D_{\xi}$ of $(D, G)$ are excellent with respect to all (respectively, solvable) coverings, then $C$ is excellent with respect to all (respectively, solvable) coverings.

Proof. By Theorem 5.1, $C(k)=\bigsqcup_{\xi} \pi_{\xi}\left(D_{\xi}(k)\right)$. Now, by Proposition 5.17,

$$
C(k) \subset C\left(\mathbb{A}_{k}\right)^{\mathrm{f}-\operatorname{cov}}=\bigcup_{\xi} \pi_{\xi}\left(D_{\xi}\left(\mathbb{A}_{k}\right)_{\bullet}^{\mathrm{f}-\operatorname{cov}}\right)=\bigcup_{\xi} \pi_{\xi}\left(D_{\xi}(k)\right)=C(k) .
$$

If $G$ is solvable, the same proof shows the statement for $C\left(\mathbb{A}_{k}\right)^{\mathrm{f}-\text {-sol }}$.

Proposition 8.5. Let $C \stackrel{\phi}{\rightarrow} X$ be a nonconstant morphism over $k$ from the curve $C$ into a variety $X$. If $X$ is excellent with respect to all, solvable, or abelian coverings, then so is $C$. In particular, if $X\left(\mathbb{A}_{k}\right)_{\bullet}^{\mathrm{f}-\mathrm{ab}}=X(k)$ and $C(k)=\varnothing$, then the absence of rational points on $C$ is explained by the Brauer-Manin obstruction.

Proof. First assume that $C$ is of genus zero. Then either $C\left(\mathbb{A}_{k}\right)_{\bullet}=\varnothing$, and there is nothing to prove, or else $C(k)$ is dense in $C\left(\mathbb{A}_{k}\right)_{\bullet}$, implying that $X(k) \subsetneq \overline{X(k)} \subset$ $X\left(\mathbb{A}_{k}\right)_{\bullet}^{\mathrm{f}-\operatorname{cov}}$ and thus contradicting the assumption.

Now assume that $C$ is of genus at least 1 . Let $P \in C\left(\mathbb{A}_{k}\right)_{\bullet}^{\mathrm{f}-\mathrm{cov} / \mathrm{f}-\mathrm{sol} / \mathrm{f}-\mathrm{ab}}$. Then by Proposition 5.9, $\phi(P) \in X\left(\mathbb{A}_{k}\right)^{\mathrm{f}-\mathrm{cov} / \mathrm{f} \text {-sol/f-ab }}=X(k)$. Let $Z \subset C$ be the preimage (subscheme) of $\phi(P) \in X(k)$ in $C$. This is finite, since $\phi$ is nonconstant. Then we have that $P$ is in the image of $Z\left(\mathbb{A}_{k}\right)_{\bullet}$ in $C\left(\mathbb{A}_{k}\right)_{0}$. Now apply Theorem 8.2 to conclude that $P \in C\left(\mathbb{A}_{k}\right)_{\bullet}^{\mathrm{f}-\mathrm{ab}} \cap Z\left(\mathbb{A}_{k}\right)_{\bullet}=Z(k) \subset C(k)$. 
As an application, we have the following.

Theorem 8.6. Let $C \rightarrow A$ be a nonconstant morphism over $k$ of a curve $C$ into an abelian variety $A$. Assume that $\amalg(k, A)_{\mathrm{div}}=0$ and that $A(k)$ is finite. (For example, this is the case when $k=\mathbb{Q}$ and $A$ is modular of analytic rank zero.) Then $C$ is excellent with respect to abelian coverings. In particular, if $C(k)=\varnothing$, then the absence of rational points on $C$ is explained by the Brauer-Manin obstruction.

Proof. By Corollary 6.2, we have $A\left(\mathbb{A}_{k}\right)_{\bullet}^{\text {f-ab }}=A(k)$. Now by Proposition 8.5 , the claim follows.

This generalizes a result proved by Siksek [2004] under additional assumptions on the Galois action on the fibers of $\phi$ above $k$-rational points of $A$, in the case that $C(k)$ is empty. A similar observation was made independently by Colliot-Thélène [2004]. Note that both previous results are in the context of the Brauer-Manin obstruction.

Examples 8.7. We can use Theorem 8.6 to produce many examples of curves $C$ over $\mathbb{Q}$ that are excellent with respect to abelian coverings. Concretely, let us look at the curves $C_{a}: y^{2}=x^{6}+a$, where $a$ is a nonzero integer. $C_{a}$ maps to the two elliptic curves $E_{a}: y^{2}=x^{3}+a$ and $E_{a^{2}}$ (the latter by sending $(x, y)$ to $\left(a / x^{2}, a y / x^{3}\right)$ ). So whenever one of these elliptic curves has (analytic) rank zero, we know that $C_{a}$ is excellent with respect to abelian coverings. For example, this is the case for all $a$ such that $|a| \leq 20$, with the exception of $a=-15,-13,-11,3,10,11,15,17$. Note that $C_{a}(\mathbb{Q})$ is always nonempty (there are two rational points at infinity).

We can even show a whole class of interesting curves to be excellent with respect to abelian coverings.

Corollary 8.8. If $C / \mathbb{Q}$ is one of the modular curves $X_{0}(N), X_{1}(N), X(N)$ and such that the genus of $C$ is positive, then $C$ is excellent with respect to abelian coverings.

Proof. By a result of Mazur [1977], every Jacobian $J_{0}(p)$ of $X_{0}(p)$, where $p=11$ or $p \geq 17$ is prime, has a nontrivial factor of analytic rank zero. Also, if $M \mid N$, then there are nonconstant morphisms $X_{1}(N) \rightarrow X_{0}(N) \rightarrow X_{0}(M)$. Hence the assertion is true for all $X_{0}(N)$ and $X_{1}(N)$ such that $N$ is divisible by one of the primes in Mazur's result. For the other minimal $N$ such that $X_{0}(N)$ (respectively, $\left.X_{1}(N)\right)$ is of positive genus, William Stein's tables [ $\geq 2007$ ] prove that there is a factor of $J_{0}(N)$ (respectively, $J_{1}(N)$ ) of analytic rank zero. So we get the result for all $X_{0}(N)$ and $X_{1}(N)$ of positive genus. Finally, $X(N)$ maps to $X_{0}\left(N^{2}\right)$, and so we obtain the result also for $X(N)$ (except in the genus zero cases $N=1,2,3,4,5$ ).

For another example, involving high-genus Shimura curves, see [Skorobogatov 2005]. 
Remark 8.9. There is some relation with the "Section Conjecture" from Grothendieck's anabelian geometry [Grothendieck 1997]. Let $C / k$ be a smooth projective geometrically connected curve of genus $\geq 2$. One can prove that if $C$ has the "section property", then $C$ is excellent with respect to all coverings, which in turn implies that $C$ has the "birational section property". See [Koenigsmann 2005] for definitions. For example, all the curves $X_{0}(N), X_{1}(N)$ and $X(N)$ have the birational section property if they are of higher genus.

\section{Discussion}

In the preceding section, we have seen that we can construct many examples of higher-genus curves that are excellent with respect to abelian coverings. This leads us to the following conjecture.

Conjecture 9.1 (Main Conjecture). If $C$ is a smooth projective geometrically connected curve over a number field $k$, then $C$ is very good.

By what we have seen, for curves of genus 1 , this is equivalent to saying that the divisible subgroup of $\amalg(k, E)$ is trivial, for every elliptic curve $E$ over $k$. For curves $C$ of higher genus, the statement means that $C$ is excellent with respect to abelian coverings. We recall that our conjecture would follow in this case from the "Adelic Mordell-Lang Conjecture" formulated in Question 3.12.

Remark 9.2. When $k$ is a global function field of characteristic $p$, then the Main Conjecture holds when $J=\operatorname{Jac}_{C}$ has no isotrivial factor and $J\left(k^{\text {sep }}\right)\left[p^{\infty}\right]$ is finite. See recent work by Poonen and Voloch [2006].

If the Main Conjecture holds for $C$ and $C(k)$ is empty, then (as previously discussed) we can find a torsor that has no twists with points everywhere locally and thus prove that $C(k)$ is empty. The validity of the conjecture (even just in case $C(k)$ is empty) therefore implies that we can algorithmically decide whether a given smooth projective geometrically connected curve over a number field $k$ has rational points or not.

In Section 7 above, we have shown that for a curve $C$, we have

$$
C\left(\mathbb{A}_{k}\right)_{\bullet}^{\mathrm{f}-\mathrm{ab}}=C\left(\mathbb{A}_{k}\right)_{\bullet}^{\mathrm{Br}},
$$

where on the right hand side, we have the Brauer subset of $C\left(\mathbb{A}_{k}\right)_{\bullet}$, that is, the subset cut out by conditions coming from the Brauer group of $C$. We say that, if $C\left(\mathbb{A}_{k}\right)_{\bullet}^{\mathrm{Br}}=\varnothing$, there is a Brauer-Manin obstruction against rational points on $C$. A corollary of our Main Conjecture is that the Brauer-Manin obstruction is the only obstruction against rational points on curves over number fields (which means that $C(k)=\varnothing$ implies $\left.C\left(\mathbb{A}_{k}\right)_{\bullet}^{\mathrm{Br}}=\varnothing\right)$. To our knowledge, before this work (and Poonen's heuristic, see his conjecture below, which was influenced by the discussions 
we had at the IHP in Paris in Fall 2004) nobody gave a conjecturally positive answer to the question, first formulated on page 133 in [Skorobogatov 2001], whether the Brauer-Manin obstruction might be the only obstruction against rational points on curves. No likely counterexample is known, but there is an ever-growing list of examples, for which the failure of the Hasse Principle could be explained by the Brauer-Manin obstruction; see the discussion below (which does not pretend to be exhaustive) or also Skorobogatov's recent paper Skorobogatov [2005] on Shimura curves.

Let $v$ be a place of $k$. Under a local condition at $v$ on a rational point $P \in C$, we understand the requirement that the image of $P$ in $C\left(k_{v}\right)$ is contained in a specified closed and open ("clopen") subset of $C\left(k_{v}\right)$. If $v$ is an infinite place, this just means that we require $P$ to be on some specified connected component(s) of $C\left(k_{v}\right)$; for finite places, we can take something like a "residue class". With this notion, the Main Conjecture above is equivalent to the following statement.

Let $C / k$ be a curve as above. Specify local conditions at finitely many places of $k$ and assume that there is no point in $C(k)$ satisfying these conditions. Then there is some $n \geq 1$ such that no point in $\prod_{v} X_{v} \subset C\left(\mathbb{A}_{k}\right)_{\bullet}$ survives the $n$-covering of $C$, where $X_{v}$ is the set specified by the local condition at those places where a condition is specified, and $X_{v}=C\left(k_{v}\right)\left(\right.$ or $\left.\pi_{0}\left(C\left(k_{v}\right)\right)\right)$ otherwise.

This says that the "finite abelian" obstruction (equivalently, the Brauer-Manin obstruction) is the only obstruction against weak approximation in $C\left(\mathbb{A}_{k}\right)$.

We see that the conjecture implies that we can decide if a given finite collection of local conditions can be satisfied by a rational point. Now the question is how practical it might be to actually do this in concrete cases. For certain classes of curves and specific values of $n$, it may be possible to explicitly and efficiently find the relevant twists. For example, this can be done for hyperelliptic curves and $n=2$; compare [Bruin and Stoll 2007a]. However, for general curves and/or general $n$, this approach is likely to be infeasible.

On the other hand, assume that we can find $J(k)$ explicitly, where $J$, as usual, is the Jacobian of $C$. This is the case (at least in principle) when $\amalg(k, J)_{\operatorname{div}}=0$. Then we can approximate $C\left(\mathbb{A}_{k}\right)_{0}^{\mathrm{f}-\mathrm{ab}}$ more and more precisely by looking at the images of $C\left(\mathbb{A}_{k}\right)_{\bullet}$ and of $J(k)$ in $\prod_{v \in S} J\left(k_{v}\right) / N J\left(k_{v}\right)$ for increasing $N$ and finite sets $S$ of places of $k$. If $C(k)$ is empty and the Main Conjecture holds, then for some choice of $S$ and $N$, the two images will not intersect, giving an explicit proof that $C(k)=$ $\varnothing$. An approach like this was proposed (and carried out for some twists of the Fermat quartic) by Scharaschkin [1999]. See [Flynn 2004] for an implementation of this method and [Bruin and Stoll 2007b] for improvements. In [Poonen et al. 2007], this procedure is used to rule out rational points satisfying certain local conditions on a genus 3 curve whose Jacobian has Mordell-Weil rank 3. 
In order to test the conjecture, Nils Bruin and the author conducted an experiment; see [Bruin and Stoll 2006]. We considered all genus 2 curves over $\mathbb{Q}$ of the form

$$
y^{2}=f_{6} x^{6}+f_{5} x^{5}+\cdots+f_{1} x+f_{0}
$$

with coefficients $f_{0}, \ldots, f_{6} \in\{-3,-2, \ldots, 3\}$. For each isomorphism class of curves thus obtained, we attempted to decide if there are rational points or not. On about 140,000 of these roughly 200,000 curves (up to isomorphism), we found a (fairly) small rational point. Of the remaining about 60,000 , about half failed to have local points at some place. On the remaining about 30,000 curves, we performed a 2-descent and found that for all but 1,492 curves $C, C\left(\mathbb{A}_{\mathbb{Q}}\right)_{\bullet}^{2-a b}=\varnothing$, proving that $C(\mathbb{Q})=\varnothing$ as well. For the 1,492 curves that were left over, we found generators of the Mordell-Weil group (assuming the Birch and Swinnerton-Dyer Conjecture for a small number of them) and then did a computation along the lines sketched above. This turned out to be successful for all curves, proving that none of them has a rational point. The conclusion is that the Main Conjecture holds for curves $C$ as in $(9-1)$ if $C(\mathbb{Q})=\varnothing$, assuming $\amalg(\mathbb{Q}, J)_{\text {div }}=0$ for the Jacobian $J$ if $C$ is one of the 1,492 curves mentioned, and assuming in addition the Birch and Swinnerton-Dyer Conjecture if $C$ is one of 42 specific curves.

At least in case $C(k)$ is empty, there are heuristic arguments due to Poonen [2006] that suggest that an even stronger form of our conjecture might be true.

Conjecture 9.3 (Poonen). Let $C$ be a smooth projective geometrically connected curve of genus $\geq 2$ over a number field $k$, and assume that $C(k)=\varnothing$. Assume further that $C$ has a rational divisor class of degree 1 , and let $\iota: C \rightarrow J$ be the induced embedding of $C$ into its Jacobian $J$. Then there is a finite set $S$ of finite places of good reduction for $C$ such that the image of $J(k)$ in $\prod_{v \in S} J\left(\mathbb{F}_{v}\right)$ does not meet $\prod_{v \in S} \iota\left(C\left(\mathbb{F}_{v}\right)\right)$.

Note that under the assumption $\amalg(k, J)_{\mathrm{div}}=0$, we must have a rational divisor (class) of degree 1 on $C$ whenever $C\left(\mathbb{A}_{k}\right)_{\bullet}^{\mathrm{f}-\mathrm{ab}} \neq \varnothing$, compare Corollary 6.6, so the condition above is not an essential restriction.

Let us for a moment assume that Poonen's Conjecture holds and that all abelian varieties $A / k$ satisfy $\amalg(k, A)_{\operatorname{div}}=0$. Then for all curves $C / k$ of higher genus, $C(k)=\varnothing$ implies $C\left(\mathbb{A}_{k}\right)_{\bullet}^{\text {f-ab }}=\varnothing$. If we apply this observation to coverings of $C$, then we find that $C$ must be excellent with respect to solvable coverings. The argument goes like this. Let $P \in C\left(\mathbb{A}_{k}\right)_{\bullet}^{\text {f-sol }}$, and assume $P \notin C(k)$. There are only finitely many rational points on $C$, and hence there is an $n$ such that $P$ lifts to a different $n$-covering $D$ of $C$ than all the rational points. (Take $n$ such that $P-Q$ is not divisible by $n$ in $J\left(\mathbb{A}_{k}\right)_{\text {. }}$, for all $Q \in C(k)$, where $J$ is the Jacobian of $C$.) In particular, $D(k)$ must be empty. But then, by Poonen's Conjecture, we have 
$D\left(\mathbb{A}_{k}\right)_{\bullet}^{\mathrm{f} \text {-ab }}=\varnothing$, so $P$ cannot lift to $D$ either. This contradiction shows that $P$ must be a rational point.

In particular, this would imply that all higher-genus curves have the "birational section property"; compare Remark 8.9.

A more extensive and detailed discussion of these conjectures, their relations to other conjectures, and evidence for them will be published elsewhere.

Acknowledgments. I would like to thank Bjorn Poonen for very fruitful discussions and Jean-Louis Colliot-Thélène, Alexei Skorobogatov, David Harari and the anonymous referee for reading previous versions of this paper carefully and making some very useful comments and suggestions. Further input was provided by Jordan Ellenberg, Dennis Eriksson, Joost van Hamel, Florian Pop and Felipe Voloch. Last but not least, thanks are due to the Centre Émile Borel of the Institut Henri Poincaré in Paris for hosting a special semester on "Explicit methods in number theory" in Fall 2004. A large part of this work has its origins in discussions I had while I was there.

\section{References}

[Breuil et al. 2001] C. Breuil, B. Conrad, F. Diamond, and R. Taylor, "On the modularity of elliptic curves over Q: wild 3-adic exercises", J. Amer. Math. Soc. 14:4 (2001), 843-939. MR 2002d:11058 Zbl 0982.11033

[Bruin and Stoll 2006] N. Bruin and M. Stoll, "Deciding existence of rational points on curves: an experiment", 2006. To appear. math.NT/0604524

[Bruin and Stoll 2007a] N. Bruin and M. Stoll, "2-cover descent on hyperelliptic curves”, 2007. In preparation.

[Bruin and Stoll 2007b] N. Bruin and M. Stoll, "The Mordell-Weil sieve: Proving nonexistence of rational points on curves", 2007. In preparation.

[Cassels and Guy 1966] J. W. S. Cassels and M. J. T. Guy, "On the Hasse principle for cubic surfaces”, Mathematika 13 (1966), 111-120. MR 35 \#2841 Zbl 0151.03405

[Chevalley and Weil 1932] C. Chevalley and A. Weil, "Un théorème d'arithmétique sur les courbes algébriques", C. R. Acad. Sci., Paris 195 (1932), 570-572. Zbl 0005.21611

[Colliot-Thélène 2004] J.-L. Colliot-Thélène, "Une remarque sur l'obstruction de Brauer-Manin sur les courbes", 2004. Email message.

[Colliot-Thélène and Sansuc 1987] J.-L. Colliot-Thélène and J.-J. Sansuc, "La descente sur les variétés rationnelles. II”, Duke Math. J. 54:2 (1987), 375-492. MR 89f:11082 Zbl 0659.14028

[Colliot-Thélène et al. 1980] J.-L. Colliot-Thélène, D. Coray, and J.-J. Sansuc, "Descente et principe de Hasse pour certaines variétés rationnelles", J. Reine Angew. Math. 320 (1980), 150-191. MR 82f:14020 Zbl 0434.14019

[Colliot-Thélène et al. 1987a] J.-L. Colliot-Thélène, D. Kanevsky, and J.-J. Sansuc, "Arithmétique des surfaces cubiques diagonales", pp. 1-108 in Diophantine approximation and transcendence theory (Bonn, 1985), edited by G. Wüstholz, Lecture Notes in Math. 1290, Springer, Berlin, 1987. MR 89g:11051 Zbl 0639.14018 
[Colliot-Thélène et al. 1987b] J.-L. Colliot-Thélène, J.-J. Sansuc, and P. Swinnerton-Dyer, "Intersections of two quadrics and Châtelet surfaces. I", J. Reine Angew. Math. 373 (1987), 37-107. MR 88m:11045a Zbl 0622.14029

[Flynn 2004] E. V. Flynn, "The Hasse principle and the Brauer-Manin obstruction for curves", Manuscripta Math. 115:4 (2004), 437-466. MR 2005j:11047 Zbl 1069.11023

[Grothendieck 1997] A. Grothendieck, "Brief an G. Faltings", pp. 49-58 in Geometric Galois actions, 1, edited by L. Schneps and P. Lochak, London Math. Soc. Lecture Note Ser. 242, Cambridge Univ. Press, Cambridge, 1997. With an English translation on pp. 285-293. MR 99c:14023 Zbl 0901.14002

[Harari 1996] D. Harari, "Obstructions de Manin transcendantes", pp. 75-87 in Number theory (Paris, 1993-1994), edited by S. David, London Math. Soc. Lecture Note Ser. 235, Cambridge Univ. Press, Cambridge, 1996. MR 99e:14025 Zbl 0926.14009

[Harari 2000] D. Harari, "Weak approximation and nonabelian fundamental groups", Ann. Sci. École Norm. Sup. (4) 33:4 (2000), 467-484. MR 2002e:14034 Zbl 1073.14522

[Harari and Skorobogatov 2002] D. Harari and A. N. Skorobogatov, "Nonabelian cohomology and rational points", Compositio Math. 130:3 (2002), 241-273. MR 2003b:11056 Zbl 1019.14012

[Harari and Skorobogatov 2005] D. Harari and A. Skorobogatov, "Nonabelian descent and the arithmetic of Enriques surfaces", Int. Math. Res. Not. 52 (2005), 3203-3228. MR 2006m:14031 Zbl 05001995

[Hartshorne 1977] R. Hartshorne, Algebraic geometry, Graduate Texts in Mathematics, No. 52, Springer, New York, 1977. MR 57 \#3116 Zbl 0367.14001

[Hrushovski 1996] E. Hrushovski, "The Mordell-Lang conjecture for function fields", J. Amer. Math. Soc. 9:3 (1996), 667-690. MR 97h:11154 Zbl 0864.03026

[Koenigsmann 2005] J. Koenigsmann, “On the 'section conjecture' in anabelian geometry”, J. Reine Angew. Math. 588 (2005), 221-235. MR 2006h:14032 Zbl 1108.14021

[Kolyvagin 1988] V. A. Kolyvagin, "Finiteness of $E(\mathbf{Q})$ and $\operatorname{SH}(E, \mathbf{Q})$ for a subclass of Weil curves", Izv. Akad. Nauk SSSR Ser. Mat. 52:3 (1988), 522-540, 670-671. MR 89m:11056 Zbl 0662.14017

[Kolyvagin and Logachëv 1989] V. A. Kolyvagin and D. Y. Logachëv, "Finiteness of the Shafarevich-Tate group and the group of rational points for some modular abelian varieties", Algebra $i$ Analiz 1:5 (1989), 171-196. MR 91c:11032 Zbl 0728.14026

[Manin 1971] Y. I. Manin, "Le groupe de Brauer-Grothendieck en géométrie diophantienne”, pp. 401-411 in Actes du Congrès International des Mathématiciens, Tome 1 (Nice, 1970), GauthierVillars, Paris, 1971. MR 55 \#356 Zbl 0239.14010

[Mazur 1977] B. Mazur, "Modular curves and the Eisenstein ideal", Inst. Hautes Études Sci. Publ. Math. 47 (1977), 33-186 (1978). MR 80c:14015 Zbl 0394.14008

[Poonen 2006] B. Poonen, "Heuristics for the Brauer-Manin obstruction for curves", Experiment. Math. 15:4 (2006), 415-420. MR 2293593 Zbl 05196200

[Poonen and Voloch 2006] B. Poonen and J. F. Voloch, "The Brauer-Manin obstruction for subvarieties of abelian varieties over function fields", preprint, 2006. math/0612263

[Poonen et al. 2007] B. Poonen, E. F. Schaefer, and M. Stoll, "Twists of $X(7)$ and primitive solutions to $x^{2}+y^{3}=z^{7}$,, Duke Math. J. 137:1 (2007), 103-158. MR 2309145 Zbl 05145500

[Scharaschkin 1999] V. Scharaschkin, Local-global problems and the Brauer-Manin obstruction, PhD Thesis, University of Michigan, 1999. 
[Serre 1971] J.-P. Serre, "Sur les groupes de congruence des variétés abéliennes. II", Izv. Akad. Nauk SSSR Ser. Mat. 35 (1971), 731-737. MR 44 \#6702 Zbl 0222.14025

[Serre 1988] J.-P. Serre, Algebraic groups and class fields, Graduate Texts in Mathematics 117, Springer, New York, 1988. Translated from the French. MR 88i:14041 Zbl 0703.14001

[Serre 2000] J.-P. Serre, Lettre à Ken Ribet du 7/3/1986, no. 138, Springer-Verlag, Berlin, 2000.

[Siksek 2004] S. Siksek, "The Brauer-Manin obstruction for curves having split Jacobians", $J$. Théor. Nombres Bordeaux 16:3 (2004), 773-777. MR 2006e:14026 Zbl 1076.14033

[Skorobogatov 1999] A. N. Skorobogatov, "Beyond the Manin obstruction", Invent. Math. 135:2 (1999), 399-424. MR 2000c:14022 Zbl 0951.14013

[Skorobogatov 2001] A. Skorobogatov, Torsors and rational points, Cambridge Tracts in Mathematics 144, Cambridge University Press, Cambridge, 2001. MR 2002d:14032 Zbl 0972.14015

[Skorobogatov 2005] A. Skorobogatov, "Shimura coverings of Shimura curves and the Manin obstruction”, Math. Res. Lett. 12:5-6 (2005), 779-788. MR 2006k:11113 Zbl 05001028

[Stein $\geq 2007$ W. W. Stein, "Modular forms database", Available at http://modular.math.washington.edu/Tables/tables.html.

[Swinnerton-Dyer 1962] H. P. F. Swinnerton-Dyer, “Two special cubic surfaces”, Mathematika 9 (1962), 54-56. MR 25 \#3413 Zbl 0103.38302

[Taylor and Wiles 1995] R. Taylor and A. Wiles, "Ring-theoretic properties of certain Hecke algebras", Ann. of Math. (2) 141:3 (1995), 553-572. MR 96d:11072 Zbl 0823.11030

[Viada 2003] E. Viada, "The intersection of a curve with algebraic subgroups in a product of elliptic curves", Ann. Sc. Norm. Super. Pisa Cl. Sci. (5) 2:1 (2003), 47-75. MR 2004c:11099 Zbl 05019603

[Voloch 1991] J. F. Voloch, "On the conjectures of Mordell and Lang in positive characteristics", Invent. Math. 104:3 (1991), 643-646. MR 92d:11067 Zbl 0735.14019

[Wang 1996] L. Wang, "Brauer-Manin obstruction to weak approximation on abelian varieties", Israel J. Math. 94 (1996), 189-200. MR 97e:11069 Zbl 0870.14032

[Wiles 1995] A. Wiles, "Modular elliptic curves and Fermat's last theorem", Ann. of Math. (2) 141:3 (1995), 443-551. MR 96d:11071 Zbl 0823.11029

Communicated by Bjorn Poonen

Received 2007-01-24 Revised 2007-10-23 Accepted 2007-11-20

m.stoll@jacobs-university.de School of Engineering and Science, Jacobs University Bremen, P.O.Box 750561, 28725 Bremen, Germany http://www.faculty.jacobs-university.de/mstoll/ 


\title{
Algebra \& Number Theory
}

www.jant.org

\section{EDITORS}

\section{MANAGING EDITOR}

Bjorn Poonen

University of California

Berkeley, USA

\author{
EDITORIAL BOARD CHAIR \\ David Eisenbud \\ University of California \\ Berkeley, USA
}

\section{BOARD OF EDITORS}

\section{Georgia Benkart}

Dave Benson

Richard E. Borcherds

John H. Coates

J-L. Colliot-Thélène

Brian D. Conrad

Hélène Esnault

Hubert Flenner

Edward Frenkel

Andrew Granville

Joseph Gubeladze

Ehud Hrushovski

Craig Huneke

Mikhail Kapranov

Yujiro Kawamata

János Kollár

Hendrik W. Lenstra

Yuri Manin

Barry Mazur
University of Wisconsin, Madison, USA

University of Aberdeen, Scotland

University of California, Berkeley, USA

University of Cambridge, UK

CNRS, Université Paris-Sud, France

University of Michigan, USA

Universität Duisburg-Essen, Germany

Ruhr-Universität, Germany

University of California, Berkeley, USA

Université de Montréal, Canada

San Francisco State University, USA

Hebrew University, Israel

University of Kansas, USA

Yale University, USA

University of Tokyo, Japan

Princeton University, USA

Universiteit Leiden, The Netherlands

Northwestern University, USA

Harvard University, USA
Susan Montgomery

Shigefumi Mori

Andrei Okounkov

Raman Parimala

Victor Reiner

Karl Rubin

Peter Sarnak

Michael Singer

Ronald Solomon

Vasudevan Srinivas

J. Toby Stafford

Bernd Sturmfels

Richard Taylor

Ravi Vakil

Michel van den Bergh

Marie-France Vignéras

Kei-Ichi Watanabe

Andrei Zelevinsky

Efim Zelmanov
University of Southern California, USA

RIMS, Kyoto University, Japan

Princeton University, USA

Emory University, USA

University of Minnesota, USA

University of California, Irvine, USA

Princeton University, USA

North Carolina State University, USA

Ohio State University, USA

Tata Inst. of Fund. Research, India

University of Michigan, USA

University of California, Berkeley, USA

Harvard University, USA

Stanford University, USA

Hasselt University, Belgium

Université Paris VII, France

Nihon University, Japan

Northeastern University, USA

University of California, San Diego, USA

\section{PRODUCTION}

ant@mathscipub.org

Paulo Ney de Souza, Production Manager

Silvio Levy, Senior Production Editor

See inside back cover or www.jant.org for submission instructions.

The subscription price for 2007 is US \$120/year for the electronic version, and \$180/year for print and electronic. Subscriptions, requests for back issues from the last three years and changes of subscribers address should be sent to Mathematical Sciences Publishers, Department of Mathematics, University of California, Berkeley, CA 94720-3840, USA.

Algebra \& Number Theory (ISSN 1937-0652) at Mathematical Sciences Publishers, Department of Mathematics, University of California, Berkeley, CA 94720-3840 is published continuously online. Periodical rate postage paid at Berkeley, CA 94704, and additional mailing offices.

ANT peer review and production are managed by EditFLOW ${ }^{\mathrm{TM}}$ from Mathematical Sciences Publishers.

\author{
PUBLISHED BY \\ mathematical sciences publishers \\ http://www.mathscipub.org \\ A NON-PROFIT CORPORATION \\ Typeset in LATEX
}

Copyright $\odot 2007$ by Mathematical Sciences Publishers 


\section{Algebra \& Number Theory}

Volume $1 \quad$ No. $4 \quad 2007$

Finite descent obstructions and rational points on curves MiCHAEL STOLL

Del Pezzo surfaces and representation theory

Vera V. Serganova and AleXei N. Skorobogatov

The zeta function of monomial deformations of Fermat hypersurfaces

REMKE KLOOSTERMAN

Dual graded graphs for Kac-Moody algebras 